\title{
Stable three generation standard-like model from a tachyonic ten dimensional heterotic-string vacuum
}

\author{
Alon E. Faraggi ${ }^{\mathrm{a}}$, Viktor G. Matyas $^{\mathrm{b}}$, Benjamin Percival $^{\mathrm{c}}$ \\ Department of Mathematical Sciences, University of Liverpool, Liverpool L69 7ZL, UK
}

Received: 3 January 2020 / Accepted: 30 March 2020 / Published online: 23 April 2020

(C) The Author(s) 2020

\begin{abstract}
Recently it was proposed that the ten dimensional tachyonic superstring vacua may serve as good starting points for the construction of viable phenomenological models. Such phenomenologically viable models enlarge the space of possible string solutions, and may offer novel insight into some of the outstanding problems in string phenomenology. In this paper we present a three generation standard-like model that may be regarded as a compactification of a ten dimensional tachyonic vacuum. We discuss the features of the model as compared to a similar model that may be regarded as compactification of the ten dimensional $S O(16) \times S O(16)$ heterotic-string. We further argue that in the four dimensional model all the geometrical moduli are fixed perturbatively, whereas the dilaton may be fixed by hidden sector non-perturbative effects.
\end{abstract}

\section{Introduction}

The heterotic-string models in the free fermionic formulation [1-3] provide a rich laboratory to develop the methodology of connecting string theory with observational data. Since the late eighties this class of string compactifications produced an abundance of three generation models [4-22], with qualitatively realistic properties, as well as an arena for investigation of cosmological scenarios [23]. The relevant class of string compactifications are $Z_{2} \times Z_{2}$ orbifolds of six dimensional toroidal manifolds [24-27], that are related to compactifications on $Z_{2}$ orbifolds of $K_{3} \times T_{2}$ surfaces. This class of internal spaces produces a rich symmetry structure also from a purely mathematical point of view [28].

\footnotetext{
a e-mail: alon.faraggi@ liv.ac.uk (corresponding author)

b e-mail: viktor.matyas@liv.ac.uk

c e-mail: benjamin.percival@liv.ac.uk
}

Since the early days of string phenomenology, the majority of studies have been devoted to the analysis of the $N=1$ supersymmetric string vacua. Supersymmetry is then broken in the effective low energy field theory limit by a gaugino or matter condensate. Electroweak radiative breaking also ensues by dimensional transmutation, and is compatible with the observed parameter space. However, while supersymmetry is an elegant theoretical construction, the fact that it is not observed below the $\mathrm{TeV}$ scale lessens some of its motivating attributes. It is therefore vital to explore alternatives from the point of view of string theory.

Investigation of non-supersymmetric string models to date were conducted by studying compactifications of the tachyon-free $S O(16) \times S O(16)$ ten dimensional heterotic string theory [29-49]. This model can be generated as an orbifold of the ten dimensional supersymmetric $E_{8} \times E_{8}$ heterotic-string, and the two vacua are connected by interpolation in a compactified dimension [31,32]. Additionally, string theory gives rise to vacua that are tachyonic in ten dimensions [29-31,33]. Recently, it was proposed in Ref. [50] that these ten dimensional string configurations may serve as viable starting points for constructing phenomenological models, and offer novel perspectives on some of the outstanding issues in string phenomenology. Tachyon-free four dimensional models may be constructed and ought to be taken on par with the non-supersymmetric $S O(16) \times S O(16)$ heterotic-string. Moreover, they may reveal alternative symmetries to those provided by spacetime supersymmetry. An example is the Massive Boson-Fermion Degeneracy of [5153]. It was demonstrated in Ref. [50] that the ten dimensional tachyonic modes may be projected out by Generalised GSO projections. A standard-like tachyon free model was presented in Ref. [50], albeit with six generations rather than three. A tachyon-free three generation model in this class is still outstanding. 
In this paper, we present such a three generation Standardlike tachyon free Model that can be regarded as a compactification of a tachyonic ten dimensional string vacuum. We discuss the spectrum of the model and its distinct features compared to the supersymmetric and non-supersymmetric models emanating from the $E_{8} \times E_{8}$ and $S O(16) \times S O(16)$ heterotic-string models. Furthermore, we argue that the internal space in our construction is entirely fixed, which follows from the fact that all the untwisted geometrical moduli are projected out in the given model. We suggest that in this model all the moduli, aside from the dilaton, are fixed perturbatively, whereas the dilaton may be fixed by a hidden sector racetrack mechanism $[54,55]$. As we elaborate in the discussion, the internal structure of the model presented here is obtained from a previously constructed supersymmetric Standard-like Model [56]. Some characteristics of the two models are consequently identical. Our present models might therefore be regarded as deformation of the supersymmetric model, which conforms with the conjecture in Ref. [50] that all $(2,0)$ string compactifications are connected via orbifolds or by interpolations.

\section{Free fermionic constructions}

In the free fermion formulation models are specified in terms of boundary condition basis vectors and one-loop Generalised GSO (GGSO) phases [1-3]. The $E_{8} \times E_{8}$ and $S O(16) \times S O(16)$ models in ten dimensions are defined in terms of a common set of basis vectors

$$
\begin{aligned}
v_{1} & =\mathbb{1}=\left\{\psi^{\mu}, \chi^{1, \ldots, 6} \mid \bar{\eta}^{1,2,3}, \bar{\psi}^{1, \ldots, 5}, \bar{\phi}^{1, \ldots, 8}\right\}, \\
v_{2} & =z_{1}=\left\{\bar{\psi}^{1, \ldots, 5}, \bar{\eta}^{1,2,3}\right\}, \\
v_{3} & =z_{2}=\left\{\bar{\phi}^{1, \ldots, 8}\right\},
\end{aligned}
$$

where we adopted the conventional notation used in the free fermionic constructions [4,8-22,57-66]. The basis vector $\mathbb{1}$ is required by the consistency rules [1-3] and generates a model with an $S O$ (32) gauge group from the Neveu-Schwarz (NS) sector. The spacetime supersymmetry generator is given by the combination

$S=\mathbb{1}+z_{1}+z_{2}=\left\{\psi^{\mu}, \chi^{1, \ldots, 6}\right\}$.

The choice of Generalised GSO phase $C\left[\begin{array}{l}z_{1} \\ z_{2}\end{array}\right]= \pm 1$ selects between the $E_{8} \times E_{8}$ or $S O(16) \times S O(16)$ heteroticstrings in ten dimensions. The relation in Eq. (2) dictates that in ten dimensions the breaking of spacetime supersymmetry is correlated with the breaking pattern $E_{8} \times$ $E_{8} \rightarrow S O(16) \times S O(16)$. Equation (2) does not hold in lower dimensions, and the two breakings are not correlated.

It is noted that in both the $E_{8} \times E_{8}$ and $S O(16) \times S O(16)$ heterotic-strings in ten dimensions, the tachyonic states are projected out. The would-be tachyon in these models are obtained from the Neveu-Schwarz (NS) sector, by acting on the right-moving vacuum with a single fermionic oscillator:

$|0\rangle_{L} \otimes \bar{\phi}^{a}|0\rangle_{R}$

where in ten dimensions $a=1, \ldots, 32$. The GSO projection induced by the $S$-vector, which is the spacetime supersymmetry generator, projects out the untwisted tachyons, producing tachyon free models in both cases. As discussed in Ref. [50] obtaining the ten dimensional tachyonic vacua in the free fermionic formulation amounts to the removal of the $S$-vector from the construction. For example, the $S O(16) \times E_{8}$ heterotic-string model in ten dimensions is generated by the basis vectors $\left\{\mathbb{1}, z_{1}\right\}$ in Eq. (1), independently of the GGSO phases. Other ten dimensional configurations can similarly be obtained by substituting the $z_{1}$ basis vectors with $z_{1}=\left\{\bar{\phi}^{1, \ldots,{ }^{4}}\right\}$ and adding similar $z_{i}$ basis vectors, with four periodic fermions, and utmost two overlapping. These vacua are connected by interpolations or orbifolds along the lines of Ref. [31], and, in general, contain tachyons in their spectrum. Our interest here is in the possibility of constructing tachyon free phenomenological vacua, starting from the tachyonic ten dimensional vacua.

As noted in the ten dimensional case, compactifications of the ten dimensional tachyonic vacua amounts to removing the vector $S$ from the set of basis vectors. In four spacetime dimensions the set $\left\{\mathbb{1}, z_{1}, z_{2}\right\}$ produces a non supersymmetric model with $S U(2)^{6} \times S O(12) \times E_{8} \times$ $E_{8}$ or $S U(2)^{6} \times S O(12) \times S O(16) \times S O(16)$. In this case the untwisted tachyonic states in general reappear. It is noted also that the left-moving vector bosons remain in the spectrum, and are projected out by the additional NAHE-set basis vectors. An alternative to removing the $S$ vector from the construction is to augment it with periodic right-moving fermions. A convenient choice is given by

$\tilde{S}=\left\{\psi^{1,2}, \chi^{1,2}, \chi^{3,4}, \chi^{5,6} \mid \bar{\phi}^{1, \ldots, 4}\right\} \equiv 1$.

In this case there are no massless gravitinos, and the untwisted tachyonic states

$|0\rangle_{L} \otimes \bar{\phi}^{1, \ldots,{ }^{4}}|0\rangle_{R}$ 
are invariant under the $\tilde{S}$-vector projection. We note that the untwisted tachyons are those that descend from the ten dimensional vacuum, hence confirming that the model can indeed be regarded as compactification of a ten dimensional tachyonic vacuum. The advantage of using the vector $\tilde{S}$ is that its projection on the chiral generation is retained, hence facilitating the construction of three generation models. In Ref. [50], a three generation model with $\tilde{S}$ was presented, which is, however, tachyonic.

Our tachyon free three generation model is constructed by using a modified NAHE-set $[67,68]$, with the $S$-vector replaced by the $\tilde{S}$-vector. We refer to it as the $\overline{\mathrm{NAHE}}$-set. The basis vectors of the $\overline{\mathrm{NAHE}}$-set are shown in Eq. (6). structure, up to some charges, i.e. the states from the sectors $S+b_{i}$ may remain massless and produce scalar states in the chiral 16-representation of $S O(10)$ [49], in NAHE based models the states from the $\tilde{S}+b_{i}$-sectors do not produce massless states.

The construction of three generation models in this class proceeds by adding three or four additional basis vectors to the $\overline{\mathrm{NAHE}}$-set. The additional basis vectors break the $S O$ (10) GUT group to one of its subgroups, and simultaneously reduce the number of chiral generations to three generations. One from each of the sectors $b_{1}, b_{2}$ and $b_{3}$. In addition to the spacetime vector bosons that produce the

\begin{tabular}{|c|c|c|c|c|c|c|c|c|c|}
\hline & $\psi^{\mu}$ & $\chi^{12}$ & $\chi^{34}$ & $\chi^{56}$ & $\bar{\psi}^{1, \ldots, 5}$ & $\bar{\eta}^{1}$ & $\bar{\eta}^{2}$ & $\bar{\eta}^{3}$ & $\bar{\phi}^{1, \ldots, 8}$ \\
\hline 1 & 1 & 1 & 1 & 1 & $1, \ldots, 1$ & 1 & 1 & 1 & 111111111 \\
\hline$\tilde{S}$ & 1 & 1 & 1 & 1 & $0, \ldots, 0$ & 0 & 0 & 0 & 000111100 \\
\hline$b_{1}$ & 1 & 1 & 0 & 0 & $1, \ldots, 1$ & 1 & 0 & 0 & 0000000000 \\
\hline$b_{2}$ & 1 & 0 & 1 & 0 & $1, \ldots, 1$ & 0 & 1 & 0 & 00000000 \\
\hline$b_{3}$ & 1 & 0 & 0 & 1 & $1, \ldots, 1$ & 0 & 0 & 1 & 00000000 \\
\hline
\end{tabular}

\begin{tabular}{c|cc|cc|cc} 
& $y^{3, \ldots, 6}$ & $\bar{y}^{3, \ldots, 6}$ & $y^{1,2}, \omega^{5,6}$ & $\bar{y}^{1,2}, \bar{\omega}^{5,6}$ & $\omega^{1, \ldots, 4}$ & $\bar{\omega}^{1, \ldots, 4}$ \\
\hline \hline $\mathbf{1}$ & $1, \ldots, 1$ & $1, \ldots, 1$ & $1, \ldots, 1$ & $1, \ldots, 1$ & $1, \ldots, 1$ & $1, \ldots, 1$ \\
$\tilde{S}$ & $0, \ldots, 0$ & $0, \ldots, 0$ & $0, \ldots, 0$ & $0, \ldots, 0$ & $0, \ldots, 0$ & $0, \ldots, 0$ \\
\hline$b_{1}$ & $1, \ldots, 1$ & $1, \ldots, 1$ & $0, \ldots, 0$ & $0, \ldots, 0$ & $0, \ldots, 0$ & $0, \ldots, 0$ \\
$b_{2}$ & $0, \ldots, 0$ & $0, \ldots, 0$ & $1, \ldots, 1$ & $1, \ldots, 1$ & $0, \ldots, 0$ & $0, \ldots, 0$ \\
$b_{3}$ & $0, \ldots, 0$ & $0, \ldots, 0$ & $0, \ldots, 0$ & $0, \ldots, 0$ & $1, \ldots, 1$ & $1, \ldots, 1$
\end{tabular}

The model generated by Eq. (6) produces some novel features. The untwisted tachyonic states are projected out by the projections of each of the basis vectors $b_{i} i=1,2,3$. Hence, the model is tachyon free. For a suitable choice of GGSO phases, the four dimensional gauge group is $S O(10) \times S O(6)^{3} \times S O(16)$. Aside from the hidden sector reduction $E_{8} \rightarrow S O(16)$, the gauge symmetry generated by (6) is identical to that generated by the NAHE-set. The novelty is in the structure of the chiral generations. Whereas in models that descend from the $S O(16) \times S O(16)$ heteroticstring the chiral generations may retain their supersymmetric four dimensional gauge group, the untwisted sector produces electroweak Higgs doublets that couple to the chiral generations from the sectors $b_{i}$ and can be used to generate fermion masses. In that respect $\overline{\mathrm{NAHE}}$-based models produce a structure which is similar to that of NAHE-based models. The caveat is that in general the NAHE-based models will be tachyonic, which stems from the proliferation of tachyon producing sectors, once the four dimensional gauge group is broken to smaller factors [49]. 
Our three generation model is constructed as a variant of the Standard-like Model published in Ref. [56]. The basis vectors that extend the $\overline{\mathrm{NAHE}}$-set and generate the Standardlike Model are given by obtained by using the basis vectors of Ref. [56], i.e. with an unmodified $S$-vector, but with the change of phases

$$
C\left[\begin{array}{l}
S \\
\beta
\end{array}\right]=C\left[\begin{array}{l}
S \\
\gamma
\end{array}\right]=-1 \rightarrow C\left[\begin{array}{l}
S \\
\beta
\end{array}\right]=C\left[\begin{array}{l}
S \\
\gamma
\end{array}\right]=+1 .
$$

\begin{tabular}{|c|c|c|c|c|c|c|c|c|c|}
\hline & $\psi^{\mu}$ & $\chi^{12}$ & $\chi^{34}$ & $\chi^{56}$ & $\bar{\psi}^{1, \ldots, 5}$ & $\bar{\eta}^{1}$ & $\bar{\eta}^{2}$ & $\bar{\eta}^{3}$ & $\bar{\phi}^{1, \ldots, 8}$ \\
\hline$\alpha$ & 0 & 0 & 0 & 0 & 11100 & 1 & 0 & 0 & 11000000 \\
\hline$\beta$ & 0 & 0 & 0 & 0 & 11100 & 0 & 1 & 0 & 00110000 \\
\hline$\gamma$ & 0 & 0 & 0 & 0 & $\frac{1}{2} \frac{1}{2} \frac{1}{2} \frac{1}{2} \frac{1}{2}$ & $\frac{1}{2}$ & $\frac{1}{2}$ & $\frac{1}{2}$ & $0000 \frac{1}{2} \frac{1}{2} \frac{1}{2} \frac{1}{2}$ \\
\hline
\end{tabular}

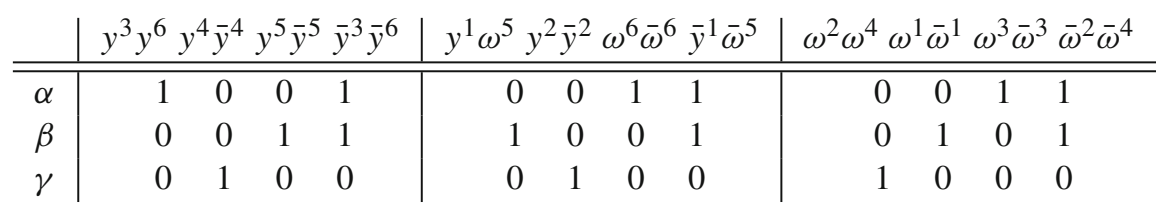

The basis vectors $\alpha, \beta$ and $\gamma$ are identical to those used in [56]. Modular invariance constraints necessitates that the GGSO phases are modified. However, the only modifications are in the phases that involve the phases associated with the basis vector $\tilde{S}$, with the choice of generalised GSO coefficients:

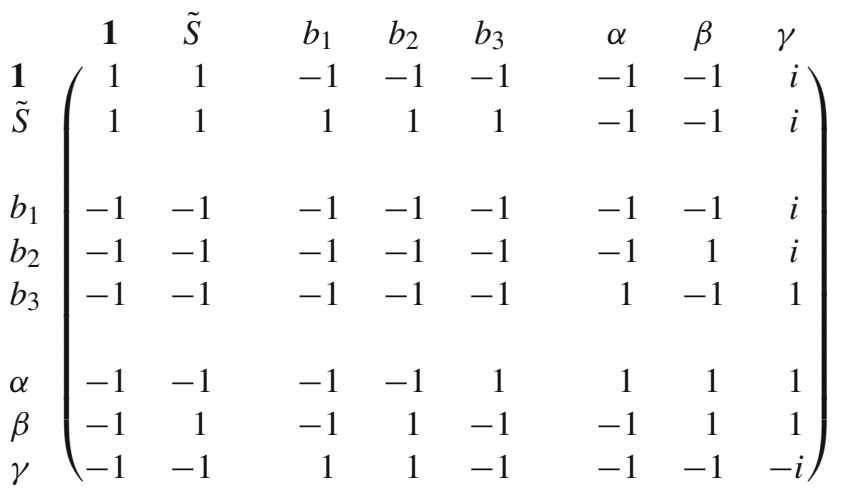

In some respects therefore the vacuum defined by Eqs. (6, 7) and (8) shares some of the properties of the model of Ref. [56]. These similarities are particularly noted with respect to the untwisted sector and the sectors $b_{1}, b_{2}$ and $b_{3}$ that produce the Standard Model spectrum. The two vacua are of course entirely different as the one in Ref. [56] is supersymmetric, whereas the one defined by Eqs. $(6,7,8)$ is not. We further remark that the model of Ref. [56] can be used to explore tachyon free compactifications of $S O(16) \times S O(16)$ heterotic string, similar to the models studied in [49]. This is
The resulting spectrum is tachyon free. With these modifications the only sectors that break supersymmetry are sectors that extend the NAHE-set. Hence, in this case the chiral matter spectrum still exhibits a supersymmetry-like structure, as discussed in [49].

Turning back to the model defined by $(6,7)$ and $(8)$, as discussed in Ref. [56], the basis vectors of the model utilise both symmetric and asymmetric boundary conditions with respect to the sets of internal worldsheet fermions $\{y \mid \bar{y}\}^{3, \ldots, 6}$, $\left\{y^{1,2}, \omega^{1,2} \mid \bar{y}^{1,2}, \bar{\omega}^{5,6}\right\}$, and $\{\omega \mid \bar{\omega}\}^{1, \ldots, 4}$. Each of these three sets is periodic in $b_{1}, b_{2}$ and $b_{3}$, respectively. This assignment induces the doublet-triplet splitting mechanism $[69,70]$ on the untwisted $5+\overline{5}$ multiplets, where symmetric assignment keeps the triplets and projects the doublets, and vice versa for the asymmetric assignment. The novelty in the model of Ref. [56], and as can be seen from Eq. (7) is that both symmetric and asymmetric boundary conditions are utilised with respect to the two sectors $b_{1}$ and $b_{2}$, whereas only asymmetric boundary conditions are utilised with respect to $b_{3}$. The result is that both the untwisted doublet and triplets, i.e. the entire $5+\overline{5}$ representations are projected from the first and second planes that couple to the states from the sectors $b_{1}$ and $b_{2}$, whereas the third untwisted plane produces a pair of electroweak Higgs doublets that couples to the states from the sector $b_{3}$ at leading order. This model, like the model of Ref. [56], contains one additional scalar Higgs doublet beyond the Standard Model. We note that the weak doublet scalar states $H_{2}, \bar{H}_{2}, H_{46}, \bar{H}_{46}, H_{47}, \bar{H}_{47}, H_{56}, \bar{H}_{56}, H_{57}, \bar{H}_{57}$ in Tables 7, 8, 9 and 10, are exotic vector-like states that should receive a heavy mass. Such states are common 
in free fermionic models. The electroweak doublet states $\tilde{h}, \overline{\tilde{h}} H_{8}, \bar{H}_{8}, H_{13}, \bar{H}_{13}$ in Tables 7 and 2 are spacetime fermionic states. Hence, the only available electroweak Higgs doublets are those arising in the NS-sector. A leading top quark Yukawa coupling is obtained at the cubic level of the potential due to the boundary condition assignment in the $\gamma$-basis vector [71,72]. An unintended consequence of these assignments is that the untwisted moduli space is reduced substantially, due to the projection of additional $S O(10)$ singlet fields from the spectrum. This led to the suggestion in Ref. [56] that all the moduli in the model, aside from the dilaton, are fixed. This structure is expected to persist in the present model.

Spacetime vector bosons in the model defined by $(6,7)$ and (8) are obtained only from the untwisted sector. The observable gauge symmetry is defined by the charges carried by the observable chiral matter states i.e. those arising from the sectors $b_{1}, b_{2}$ and $b_{3}$. It is given by:

$$
\begin{aligned}
& S U(3)_{C} \times S U(2)_{L} \times U(1)_{C} \times U(1)_{L} \times U(1)_{1,2,3} \\
& \quad \times U(1)_{4,5,6} .
\end{aligned}
$$

where,

$$
\begin{aligned}
& U(1)_{C}=\operatorname{Tr} U(3)_{C} \Rightarrow Q_{C}=\sum_{i=1}^{3} Q\left(\bar{\psi}^{i}\right), \\
& U(1)_{L}=\operatorname{Tr} U(2)_{L} \Rightarrow Q_{L}=\sum_{i=4}^{5} Q\left(\bar{\psi}^{i}\right) .
\end{aligned}
$$

The flavour $U(1)_{1,2,3}$ are generated by the worldsheet complex fermions $\bar{\eta}^{1,2,3}$ whereas $U(1)_{4,5,6}$ are generated by $\bar{\zeta}^{1,2,3}$. The complex fermions $\bar{\zeta}^{i}$ are defined as $\bar{\zeta}^{1}=$ $(1 / \sqrt{2})\left(\bar{y}^{3}+\bar{y}^{6}\right), \bar{\zeta}^{2}=(1 / \sqrt{2})\left(\bar{y}^{1}+\bar{\omega}^{5}\right)$ and $\bar{\zeta}^{3}=$ $(1 / \sqrt{2})\left(\bar{\omega}^{2}+\bar{\omega}^{4}\right)$. Each of the sectors $b_{1}, b_{2}$ and $b_{3}$ is charged with respect to $U(1)_{i}$ and $U(1)_{i+3}$. The appearance of the additional $U(1)_{4,5,6}$ symmetries arises due to the use of asymmetric boundary conditions that are essential for fixing the geometrical moduli [73]. We note that this structure of the observable gauge symmetries is similar to that of the Standard-like Models in [10-12].

The hidden sector of the model arises from the complex worldsheet fermions $\bar{\phi}^{1, \ldots, 8}$ and is given by

$S U(2)_{1, \ldots, 6} \times U(1)_{7,8}$,

where $U(1)_{7,8}$ symmetries correspond to the combinations for worldsheet charges

$$
Q_{7}=\sum_{i=5}^{6} Q\left(\bar{\phi}^{i}\right) \text { and } \quad Q_{8}=\sum_{i=7}^{8} Q\left(\bar{\phi}^{i}\right) .
$$

In NAHE-based models, the vector combination

$\zeta=\mathbb{1}+b_{1}+b_{2}+b_{3}$,

may give additional spacetime vector bosons that enhance the hidden sector gauge gauge, which are, however, projected out in the model defined by Eqs. $(6,7)$ and $(8)$, and the hidden sector is not enhanced. The retention/projection of the enhancing states from the $\zeta$-sector correspond to the $x$-map of Refs. [74,75]. The hidden sector gauge group differs from that of Ref. [56] due to the right-moving periodic fermions in the $\tilde{S}$-vector. The Neveu-Schwarz sector produces in addition to the graviton, dilaton, antisymmetric tensor and spacetime vector bosons, one pair of electroweak Higgs doublets $h^{3}$ and $\bar{h}^{3}$; six pairs of $S O(10)$ singlet fields, which are charged with respect to $U(1)_{4,5,6}$; and three fields that are neutral under the entire four dimensional gauge. These NS scalar fields are the same as those that are obtained in the model of Ref. [56]. The two model differ in the fermionic spectrum generated in the $S$ - and $\tilde{S}$-sectors, respectively, and in any combination that contains these vectors, on which we elaborate below. The full massless spectrum of the model is presented in Appendix A. All sectors, fermonic and bosonic, have CPT conjugates that are not displayed explicitly in the tables in Appendix A.

\subsection{Analysis of the spectrum}

As mentioned, the model under investigation takes the model of [56] and transforms $S$ to $\tilde{S}$ and applies the phase changes in Eq. (9). The states in the Hilbert space of this model are presented in Appendix A. It is worth exploring further the action of $\tilde{S}$-map on sectors in this model and how it differs from the SUSY generator, which is induced by the $S$-map. In supersymmetric vacua the superpartners of the states from a given sector $\rho$ are obtained from the sector $S+\rho$. In nonsupersymmetric models in which supersymmetry is broken by a GGSO phase [49], the states from the sector $S+\rho$ may be projected out, but more generally they remain in the Hilbert space with modified charges. Hence, these sectors retain the Fermi-Bose degeneracy of the massless states. Additionally, in such models, in general, there are sectors for which the states in the sector $S+\rho$ are massive. These sectors therefore do not preserve the Fermi-Bose degeneracy. Additionally, the $\zeta$-sector induces the $\zeta$-map. In models with enhanced hidden sector gauge group, the $\zeta$-mapped sectors $\rho+\zeta$, complements the states from a sector $\rho$ to representations of the enhanced symmetry group. In models in which the enhancing states are projected out, the states will be mapped to other representations. However, in many cases the number of states is preserved overall. This phenomenon was observed e.g. in the case of spinor-vector duality of Refs. [62-66,76] under the $x$-map and reflects the modular properties of the underlying partition function. Similar properties may there- 
fore be exhibited in the models with broken supersymmetry that reflect their underlying modular properties. Due to the assignment of Ramond boundary conditions to $\bar{\phi}^{3456}$ in $\tilde{S}$ there are many sectors such as those listed in Tables 9, 10, 3 and 5 for this model, where no $\tilde{S}$-mapped states appear in the spectrum. The most common reason for this is that the $\tilde{S}$-mapped sector gains additional contributions to the mass formula on the right due to the addition of the Ramond $\bar{\phi}^{3456}$ in $\tilde{S}$, thus making the $\tilde{S}$-mapped sector massive. The most notable sectors of this type are the $b_{i} i=1,2,3$ sectors that produce the three chiral generations, one from each of the sectors $b_{1}, b_{2}$ and $b_{3}$, whereas the bosonic states in the sectors $\tilde{S}+b_{i} i=1,2,3$ are massive. This should be contrasted with the model generated by employing the phase modification in Eq. (9), which breaks supersymmetry, but retains the scalar states from the sectors $S+b_{i} i=1,2,3$. However, the sector $1+\tilde{S}+b_{3}+\alpha+\beta$ shown in Table 4 , is projected out by the GGSO projections from the $\overline{\text { NAHE}}$-basis, despite giving rise to massless states a priori. In NAHE-based models such a projection of a super-partnered state cannot occur. We also note the existence of fermionic states in the $\tilde{S}$-sector that transform as doublets of the electroweak symmetry and an hidden $S U$ (2) gauge group suggesting the possible implementation of electroweak symmetry breaking by fermion condensates.

The sectors shown in Table 6 exhibit the mapping property mentioned above. The left-moving sector of the $\tilde{S}$-vector is the same as that of the $S$-vector. Hence, the $\tilde{S}$-vector still maps spacetime fermions to spacetime bosons. The $2 \gamma$-map correspond to the $x$-map of Refs. [74,75], which is a map between the spinorial sectors $b_{j}, j=1,2,3$, to the vectorial producing sectors $b_{j}+2 \gamma$ (or $b_{j}+x$ ). The $\zeta$-map then correspond to the map to sectors that supplement the hidden sector representations when the hidden sector gauge symmetry is enhanced, and otherwise maps to other states that descend from the massive spectrum. The states in these sectors are therefore arranged in three groups of four. Understanding the detail structure of the spectrum is crucial not only for understanding the properties of a single model, but rather in order to understand the global structure that underlies the larger space of vacua, as demonstrated by the spinor-vector duality [62-66,76]. Another observation of the $\tilde{S}$-map is that for certain sectors the addition of the Ramond $\bar{\phi}^{3456}$ can change the mass formula on the right so as to map a spinorial sector to a vectorial one. Such an outcome is observed, for example, in sector $b_{3} \pm \gamma$ which is spinorial, whereas $\tilde{S}+b_{3} \pm \gamma$ is vectorial.

As is common in $(2,0)$ heterotic-string compactifications [77], the model generated by the basis vectors in $(6,7)$ and GGSO phases in (8) contains six $U(1)$ symmetries with nonvanishing trace,

$$
\begin{aligned}
& \operatorname{Tr} U(1)_{1}=\operatorname{Tr} U(1)_{2}=\operatorname{Tr} U(1)_{3}=-24 \\
& \operatorname{Tr} U(1)_{4}=\operatorname{Tr} U(1)_{5}=\operatorname{Tr} U(1)_{6}=-12 .
\end{aligned}
$$

Five combinations of these $U(1)$ 's are anomaly free and one combination, given by

$$
\begin{aligned}
U(1)_{A}= & 2\left(U(1)_{1}+U(1)_{2}+U(1)_{3}\right) \\
& +\left(U(1)_{4}+U(1)_{5}+U(1)_{6}\right),
\end{aligned}
$$

remains anomalous. The anomalous $U(1)$ is removed by the Green-Schwarz-Dine-Seiberg-Witten mechanism [78,87], but gives rise to a tadpole diagram in string perturbation theory at one-loop order [79], which reflects the instability of the string vacuum. The mismatch between the bosonic and fermionic states at different mass levels produces a nonvanishing vacuum energy, which similarly gives rise to a tadpole diagram, indicating the instability of the string vacuum. We may contemplate the possibility of suppressing one against the other so that they conspire to cancel. The anomalous $U(1)$ contribution is proportional to the trace over the massless fermionic states and the sign can be altered by the GGSO projections [77,79]. Both the anomalous $U(1)$ and the vacuum energy will depend on further details of the model, which are very complicated, e.g. the potential of the remaining scalar fields in the spectrum, that can shift the vacuum. A comprehensive analysis is beyond our scope here, and possibly out of reach in terms of the contemporary tools available due to the large number of fields in our quasi-realistic model. However, we note that the same issues also plague the supersymmetric vacua with an anomalous $U(1)$ as well as vacua that are compactifications of the nonsupersymmetric $S O(16) \times S O(16)$ heterotic-string. Therefore, a shift of the vacuum is either legitimate, or illegitimate, in both cases. Any statement about the stability of the string vacua is at best speculative. We therefore propose that all of the non-tachyonic string vacua should be considered on equal footing. We can compactify the different ten dimensional vacua on the same internal structure and try to learn from the properties at the different limits. In the next section we illustrate this with regard to the question of stability of the model.

\section{Moduli fixing}

Next we discuss the question of the moduli in the string model defined by Eqs. (7) and (8). The issue of moduli in string compactification is intricate. It is only properly understood in compactifications with $(2,2)$ worldsheet supersymmetry and with at least $N=1$ spacetime supersymmetry [80], but not in the more generic $(2,0)$ compactifications. Nevertheless, we can borrow from the terminology in those cases. The fact that supersymmetric and non-supersymmetric string vacua can be interpolated $[32,47,48]$, suggest that the moduli in the more generic compactifications with $(2,0)$ worldsheet supersymmetry can be interpolated to the fields 
in the corresponding $(2,2)$ compactifications [50]. To study the moduli in the model defined by Eqs. (7) and (8) we follow the discussion in Ref. [73]. The geometrical moduli in the model are identified in terms of worldsheet Thirring interactions [84-86] that are invariant under the fermionic transformation properties defined by a given set of basis vectors, and are parameterised by untwisted fields in the massless string spectrum [73]. For symmetric orbifold models, the exactly marginal operators associated with the untwisted moduli fields have the general form $\partial X^{I} \bar{\partial} X^{J}$, where $X^{I}$, $I=1, \ldots, 6$, are the coordinates of the six-torus $T^{6}$. The untwisted moduli fields in this models admit the geometrical interpretation of background fields, which appear as couplings of the exactly marginal operators in the non-linear sigma model action. The untwisted moduli scalar fields are the background fields that are compatible with the orbifold symmetry. In the fermionic formalism the exactly marginal operators are given in terms of Abelian Thirring operators of the form $J_{L}^{i}(z) \bar{J}_{R}^{j}(\bar{z})$, where $J_{L}^{i}(z), \bar{J}_{R}^{j}(\bar{z})$ are some left- and right-moving $U(1)$ chiral currents described by worldsheet fermions. The untwisted moduli correspond to the Abelian Thirring interactions that are compatible with the GGSO projections induced by the boundary condition basis vectors, in a given string model.

The set of Abelian Thirring operators, and untwisted moduli fields, is restricted by the projections induced by progressive boundary condition basis vectors. The minimal basis set in the model defined by Eqs. $(7,8)$ contains the two vectors $\{\mathbb{1}, \tilde{S}\}$. This set generates a non-supersymmetric tachyonic model with $S O(36) \times S O(8)$ right-moving gauge group. The tachyonic states are the surviving untwisted tachyonic states in Eq. (5). As in the ten dimensional tachyon free vacua, the six $\chi_{I}$ are identified with the fermionic superpartners of the six bosonic coordinates. This is because the $\tilde{S}$-vector preserves the left-moving structure of the $S$-vector in the corresponding non-tachyonic vacua. Each pair $\left\{y^{i}, \omega^{i}\right\}$ is identified with the fermionised version of the corresponding leftmoving bosonic coordinate $X^{i}$, i.e. $i \partial X_{L}^{i} \sim y^{i} \omega^{i}$. The two dimensional action of the Abelian worldsheet Thirring interactions is

$S=\int d^{2} z h_{i j}(X) J_{L}^{i}(z) \bar{J}_{R}^{j}(\bar{z})$

where $J_{L}^{i}(i=1, \ldots, 6)$ are the left-moving chiral currents of $U(1)^{6}$ and $\bar{J}_{R}^{j}(j=1, \ldots, 22)$, are the right-moving chiral currents $U(1)^{22}$. The couplings $h_{i j}(X)$, as functions of the spacetime coordinates $X^{\mu}$, are four dimensional scalar fields that are identified with the untwisted moduli fields. In the model with the two basis vectors $\{1, \tilde{S}\}$ the $6 \times 22$ fields $h_{i j}(X)$ in Eq. (18) correspond to the 21 and 15 components of the background metric $G_{I J}$ and antisymmetric tensor $B_{I J}(I, J=1, \ldots, 6)$, plus the $6 \times 16$ Wilson lines $A_{I a}$. The $h_{i j}(X)$ fields parameterise the $S O(6,22) / S O(6) \times$
$S O$ (22) coset-space of the toroidally compactified space. The $h_{i j}$ untwisted moduli fields arise from the NS sector,

$\left|\chi^{I}\right\rangle_{L} \otimes\left|\bar{\Phi}^{+J} \bar{\Phi}^{-J}\right\rangle_{R}$,

given in terms of the 22 complex right-handed world-sheet fermions $\bar{\Phi}^{+J}$ and their complex conjugates $\bar{\Phi}^{-J}$. The corresponding marginal operators are given as

$J_{L}^{i}(z) \bar{J}_{R}^{j}(\bar{z}) \equiv: y^{i}(z) \omega^{i}(z)(z):: \bar{\Phi}^{+j}(\bar{z}) \bar{\Phi}^{-j}(\bar{z}):$.

It is seen that the transformation properties of $\chi^{i}$, which appear in the moduli (19), are the same as those of $y^{i} \omega^{i}$, which appear in the Abelian Thirring interactions (20). We further note that the supersymmetric vacuum defined by $\{\mathbb{1}, S\}$ and the non-supersymmetric vacuum defined by $\{\mathbb{1}, \tilde{S}\}$ can be connected by continuous interpolations, by turning on the appropriate Wilson lines, as demonstrated in the corresponding ten dimensional cases [31]. The important observation is that the modification of the basis vector $S \rightarrow \tilde{S}$ does not affect the untwisted scalar moduli space which is therefore identical in the two vacua, as well as in the corresponding non-supersymmetric model that descends from the $S O(16) \times S O(16)$ heterotic-string in ten dimensions.

The ensuing analysis of the untwisted moduli follows closely that of Refs. [56,73]. Adding the basis vectors $b_{1}$, $b_{2}$ and $b_{3}$, reduces the space of untwisted moduli scalars to the three sets

$h_{i j}=\left|\chi^{i}\right\rangle_{L} \otimes\left|\bar{y}^{j} \bar{\omega}^{j}\right\rangle_{R}=\left\{\begin{array}{l}(i, j=1,2) \\ (i, j=3,4) \\ (i, j=5,6)\end{array}\right.$,

that parameterise the moduli space

$\mathcal{M}=\left(\frac{S O(2,2)}{S O(2) \times S O(2)}\right)^{3}$.

These untwisted moduli fields are present in all symmetric $Z_{2} \times Z_{2}$ orbifolds. In complexified form they correspond to three Kähler and three complex structures of the $Z_{2} \times Z_{2}$ orbifold [73]. As in the case of the model of [56] The addition of the three basis vectors beyond the NAHE-set results in the projection of all the states in Eq. (21), i.e. all of the geometrical moduli are projected out. One can further check that the scalar states arising from the NS-sector are indeed identical in the two models. It should be emphasised that this outcome is particular to the boundary condition assignment for the set of left-moving real fermions $\{y, \omega\}^{1, \ldots, 6}$ and their specific pairings [73]. The basic result is that due to this particular assignment all the internal circles of the six dimensional torus are shifted asymmetrically, hence fixing the moduli of all six circles simultaneously, which is possible only in the case of the $Z_{2} \times Z_{2}$ orbifold. 
$Z_{2} \times Z_{2}$ orbifold models also contain moduli from the twisted sectors. It was argued in [73] that these moduli are also projected out from the massless spectrum in our string model. In the supersymmetric vacua this follows from the reduction of $E_{8} \times E_{8} \rightarrow S O(16) \times S O(16)$ by the basis vector set $\left\{\mathbb{1}, S, \zeta_{1}=\mathbb{1}+b_{1}+b_{2}+b_{3}, 2 \gamma\right\}$. To identify the twisted moduli in the fermionic $Z_{2} \times Z_{2}$ orbifolds, it is instrumental to consider the set $\left\{\mathbb{1}, S, \zeta_{1}, x\right\}$, with $x=\left\{\bar{\psi}^{1, \ldots, 5}, \bar{\eta}^{1,2,3}\right\}[74,75]$. The $Z_{2} \times Z_{2}$ orbifold in the $E_{8} \times E_{8}$ case reduces the observable $E_{8}$ symmetry to $E_{6} \times U(1)^{2}$, and produces states in the 27 representation of $E_{6}$ from the twisted sectors. Under the decomposition of $E_{6} \rightarrow S O(10) \times U(1)$, the 27 multiplet split as $16_{1 / 2}+10_{-1}+1_{2}$, in a convenient normalisation of the $U(1)$ generator. In the free fermionic construction the 16 spinorial components are obtained from the sectors $b_{i}$, whereas the $10+1$ components are obtained from the sectors $b_{i}+x$. The sectors $b_{i}+x$ produce an additional $E_{6}$ singlet field, which is identified with the twisted moduli [73-75]. The class of vacua with enhanced $E_{6}$ symmetry possess $(2,2)$ worldsheet supersymmetry. In vacua in which the $E_{6}$ symmetry is reduced to $S O(10) \times U(1)$, the $(2,2)$ worldsheet supersymmetry is reduced to $(2,0)$. The states from the sectors $b_{i}+x$ are mapped to hidden sector matter states [73-75], i.e. the twisted moduli are projected out. The only states that arise from the twisted sectors in this case are the observable and hidden sector matter states. These states have superpotential mass terms and therefore should not be identified as moduli. It should be emphasised, though, that any discussion of the twisted moduli in the $(2,0)$ vacua should be taken with a grain of salt, as their proper identification is only possible in vacua with $(2,2)$ worldsheet supersymmetry [8083].

More to the point, however, is the analysis of supersymmetric flat directions that was carried out in Ref. [56]. It was observed there that a certain class of flat directions, which are designated as "stringent flat directions", do not exist in the model analysed in [56]. It was further argued that "stringent flat directions" are the only flat directions that are exact to any order in the superpotential, and that non-stringent flat directions must be broken at some order. In that case, it was argued that all the moduli in the model are fixed, where the geometrical moduli are fixed by the asymmetric boundary conditions, whereas the supersymmetric moduli are fixed by the absence of exact supersymmetric flat directions, and the dilaton may be fixed by hidden sector non-perturbative effects [56]. It was further argued that supersymmetry is broken pertubatively in the model due to the existence of an anomalous $U(1)$ symmetry that produces a Fayet-Iliopolous term at one-loop [87].

As discussed above, in respect to geometrical moduli, the supersymmetric model of [56] and the non-supersymmetric model discussed here, are identical, as the map $S \rightarrow \tilde{S}$ does not affect the geometrical moduli. Borrowing from the discussion of the absence of flat directions in the supersymmetric case, we argue that also in the present vacuum all the moduli are fixed. The argument is that the internal space in the two vacua is identical and is not affected by the map $S \rightarrow \tilde{S}$, as can be seen from the assignment of the remaining boundary condition basis vectors in Eq. (7) and in Ref. [56].

We note here that in general in non-supersymmetric string vacua one expects that at a certain order in perturbation theory manifest supersymmetry breaking is also communicated to the scalar potential, lifting any flat directions. However, there are several caveats to this expectation. In the first place, the models are connected by deformations to points in the moduli space that admit tachyonic states. It is therefore not entirely clear that a given non-supersymmetric string model can stabilise at a finite value of the moduli. In the same vein, stabilisation of the moduli is a dynamical problems involving a large number of scalar fields. Whether or not all the moduli can stabilise at a finite value is a very hard problem that occupies much of the discourse in string phenomenology over the past two decades, and is still on going. Our argument here does not rely on such considerations. In our string model the geometrical moduli are simply projected out. The model is therefore by construction not connected to any points in the moduli space that admits tachyonic states. Similarly, the geometric moduli are not merely stabilised. They are frozen. Our model therefore provides an example of non-supersymmetric string vacua in which all the moduli, aside from the dilaton, are fixed, irrespective of the dynamics of vacuum stabilisation. Whether the vacuum itself is stable hinges on the possibility of stabilising the dilaton at finite value, and with positive vacuum energy. We alluded here to the possibility of using the racetrack mechanism [54,55] to stabilise the dilaton. The question of the vacuum energy in the class pertaining models will be reported in a future publication. However, we should stress that we regard our model as exploratory, providing some insight into novel possibilities in string phenomenology, rather than aiming to address the full set of questions involved. Even if one could demonstrate that a non-supersymmetric stable string model with suppressed positive vacuum energy exists at oneloop, its viability at higher order in string perturbation theory will still be open, unlike the case for supersymmetric vacua.

\section{Discussion and conclusion}

In this paper we presented a tachyon free three generation standard-like model that may be regarded as a compactification of a tachyonic ten dimensional vacuum. The model is non-supersymmetric and tachyon-free. It repre- 
sents a new class of phenomenological string vacua, with notable differences compared to vacua that can be built on the same internal structure. In this example, we considered the supersymmetric compactification of Ref. [56] as well as the construction of a model in which supersymmetry is broken by GGSO phase, á la Ref. [49]. This nonsupersymmetric version can be regarded as descending from the non-supersymmetric $S O(16) \times S O(16)$ heterotic-string in ten dimensions. Both this non-supersymmetric vacuum, as well as the supersymmetric model of Ref. [56], utilise the same set of boundary condition basis vectors with the substitution $\tilde{S} \rightarrow S$. In all three cases the untwisted NS scalar spectrum is the same, indicating that the internal structure in all three models is identical. The twisted spectrum is, however, entirely different. In the model presented here the states from the sectors $\tilde{S}+b_{j}$ are massive, whereas in the other non-supersymetric model the corresponding states from the sectors $S+b_{j}$ remain in the massless spectrum, albeit with modified $U(1)$ charges. Hence in this case the chiral generations still exhibit a supersymmetric like structure, although they do not reside in super-multiplets. These examples illustrate how compactifications of the different ten dimensional vacua can be used to explore the phenomenological properties on the same internal structure. In that respect compactifications of the tachyonic ten dimensional vacua may reveal new insight into outstanding issues in string phenomenology.

Furthermore, as the internal structure of the model was adopted from the previously constructed supersymmetric model in Ref. [56], we used the observation made there in regard to the absence of stringent flat direction, and consequently the fixing of all moduli, to argue that in our present model all moduli, aside from the dilaton, are also fixed perturbatively, whereas the dilaton may be fixed by hidden sector non-perturbative dynamics. In Ref. [56] it was argued that the absence of exact flat directions suggests that supersymmetry is broken perturbatively in the model due to the existence a Fayet-Iliopoulos term at one-loop. In our present model supersymmetry is broken explicitly at the Planck scale, but we carried forward the argument from Ref. [56] to propose that all the moduli in the current model are also fixed and hence the vacuum would be stable. We should warn, however, that any discussion of stability in non-supersymmetric string vacua is speculative, fraught with uncertainty, and possibly premature. Nevertheless, we note that the conditions that enable us to speculate on this stability are very particular to the configuration exhibited in this particular class of standard-like models. For example it was observed in Ref.
[88] that in flipped $S U(5)$ string vacua with internal structure similar to the one used in Ref. [56], there do exist stringent supersymmetric flat directions. The absence of stringent flat directions is therefore specific to vacua with similar internal structure and standard-like model gauge group. Similarly, as noted in Ref. [73] the projection of the untwisted geometrical moduli is specific to the pairing of the internal worldsheet fermions employed in our model. In this regard, we note that while the standard-like model in Refs. [5-7] shares many of the phenomenological characteristics with the standard-like model of Refs. [10-12], whereas in the later case the geometrical moduli are fixed, in the former case they are not. It is seen again that this property is specific to a particular configuration and is not generic. We may infer conservatively that the stability issue of the string vacuum can only be addressed in the very specific string vacua that come close to describing the Standard Model, rather than in the generic cases.

Acknowledgements AEF would like to thank the Weizmann Institute for hospitality, where part of this work was conducted. The work of VGM is supported in part by EPSRC grant EP/R513271/1. The work of BP is supported in part by STFC grant ST/N504130/1.

Data Availability Statement This manuscript has no associated data or the data will not be deposited. [Authors' comment: The research area of this paper is theoretical. It relates to the general data provided by the particle Standard Model rather than those a specific particle experiment.]

Open Access This article is licensed under a Creative Commons Attribution 4.0 International License, which permits use, sharing, adaptation, distribution and reproduction in any medium or format, as long as you give appropriate credit to the original author(s) and the source, provide a link to the Creative Commons licence, and indicate if changes were made. The images or other third party material in this article are included in the article's Creative Commons licence, unless indicated otherwise in a credit line to the material. If material is not included in the article's Creative Commons licence and your intended use is not permitted by statutory regulation or exceeds the permitted use, you will need to obtain permission directly from the copyright holder. To view a copy of this licence, visit http://creativecomm ons.org/licenses/by/4.0/.

Funded by $\mathrm{SCOAP}^{3}$.

\section{A The spectrum of the model}

The following tables present the spectrum of the model given in Sect. 2. All charges are multiplied by four and the CPT conjugates are omitted for all states. Throughout the tables we will make use of the vector combination: $\zeta=1+b_{1}+$ $b_{2}+b_{3}=\left\{\bar{\phi}^{1, \ldots, 8}\right\}$.

See Tables 1, 2, 3, 4, 5, 6, 7, 8, 9 and 10. 
Table 1 The untwisted Neveu-Schwarz scalar states. All charges are multipled by 4

\begin{tabular}{|c|c|c|c|c|c|c|c|c|c|c|c|c|c|c|}
\hline $\mathrm{F}$ & Sector & Name & $(C, L)$ & $Q_{C}$ & $Q_{L}$ & $Q_{\bar{\eta}^{1}}$ & $Q_{\bar{\eta}^{2}}$ & $Q_{\bar{\eta}^{3}}$ & $Q_{\bar{y} 3,6}$ & $Q_{\bar{y}^{1} \bar{w}^{5}}$ & $Q_{\bar{w}^{2,4}}$ & $S U(2)_{1, \ldots, 6}$ & $Q_{7}$ & $Q_{8}$ \\
\hline \multirow[t]{15}{*}{$\mathrm{b}$} & \multirow[t]{15}{*}{ NS } & (h) & $(1,2)$ & 0 & -4 & 0 & 0 & 4 & 0 & 0 & 0 & $(1,1,1,1,1,1)$ & 0 & 0 \\
\hline & & $(\bar{h})$ & $(1,2)$ & 0 & 4 & 0 & 0 & -4 & 0 & 0 & 0 & $(1,1,1,1,1,1)$ & 0 & 0 \\
\hline & & $\left(\Phi_{56}\right)$ & $(1,1)$ & 0 & 0 & 0 & 0 & 0 & 4 & 4 & 0 & $(1,1,1,1,1,1)$ & 0 & 0 \\
\hline & & $\left(\bar{\Phi}_{56}\right)$ & $(1,1)$ & 0 & 0 & 0 & 0 & 0 & -4 & -4 & 0 & $(1,1,1,1,1,1)$ & 0 & 0 \\
\hline & & $\left(\Phi_{56}^{\prime}\right)$ & $(1,1)$ & 0 & 0 & 0 & 0 & 0 & -4 & 4 & 0 & $(1,1,1,1,1,1)$ & 0 & 0 \\
\hline & & $\left(\bar{\Phi}_{56}^{\prime}\right)$ & $(1,1)$ & 0 & 0 & 0 & 0 & 0 & 4 & -4 & 0 & $(1,1,1,1,1,1)$ & 0 & 0 \\
\hline & & $\left(\Phi_{46}\right)$ & $(1,1)$ & 0 & 0 & 0 & 0 & 0 & 4 & 0 & 4 & $(1,1,1,1,1,1)$ & 0 & 0 \\
\hline & & $\left(\bar{\Phi}_{46}\right)$ & $(1,1)$ & 0 & 0 & 0 & 0 & 0 & -4 & 0 & -4 & $(1,1,1,1,1,1)$ & 0 & 0 \\
\hline & & $\left(\Phi_{46}^{\prime}\right)$ & $(1,1)$ & 0 & 0 & 0 & 0 & 0 & -4 & 0 & 4 & $(1,1,1,1,1,1)$ & 0 & 0 \\
\hline & & $\left(\bar{\Phi}_{46}^{\prime}\right)$ & $(1,1)$ & 0 & 0 & 0 & 0 & 0 & 4 & 0 & -4 & $(1,1,1,1,1,1)$ & 0 & 0 \\
\hline & & $\left(\Phi_{45}\right)$ & $(1,1)$ & 0 & 0 & 0 & 0 & 0 & 0 & 4 & 4 & $(1,1,1,1,1,1)$ & 0 & 0 \\
\hline & & $\left(\bar{\Phi}_{45}\right)$ & $(1,1)$ & 0 & 0 & 0 & 0 & 0 & 0 & -4 & -4 & $(1,1,1,1,1,1)$ & 0 & 0 \\
\hline & & $\left(\Phi_{45}^{\prime}\right)$ & $(1,1)$ & 0 & 0 & 0 & 0 & 0 & 0 & -4 & 4 & $(1,1,1,1,1,1)$ & 0 & 0 \\
\hline & & $\left(\bar{\Phi}_{45}^{\prime}\right)$ & $(1,1)$ & 0 & 0 & 0 & 0 & 0 & 0 & 4 & -4 & $(1,1,1,1,1,1)$ & 0 & 0 \\
\hline & & $\left(\xi_{1,2,3}\right)$ & $(1,1)$ & 0 & 0 & 0 & 0 & 0 & 0 & 0 & 0 & $(1,1,1,1,1,1)$ & 0 & 0 \\
\hline
\end{tabular}

Table 2 The $\tilde{S}$ and $\tilde{S}+\xi$ sectors. All charges are multipled by 4 and the combination $\zeta=1+b_{1}+b_{2}+b_{3}$ has been used

\begin{tabular}{|c|c|c|c|c|c|c|c|c|c|c|c|c|c|c|}
\hline $\mathrm{F}$ & Sector & Name & $(C, L)$ & $Q_{C}$ & $Q_{L}$ & $Q_{\bar{\eta}^{1}}$ & $Q_{\bar{\eta}^{2}}$ & $Q_{\bar{\eta}^{3}}$ & $Q_{\bar{y}, 6}$ & $Q_{\bar{y}^{1} \bar{w}^{5}}$ & $Q_{\bar{w}^{2,4}}$ & $S U(2)_{1, \ldots, 6}$ & $Q_{7}$ & $Q_{8}$ \\
\hline \multirow[t]{6}{*}{ f } & \multirow[t]{6}{*}{$\tilde{S}$} & $\tilde{h}$ & $(1,2)$ & 0 & 4 & 0 & 0 & 0 & 0 & 0 & 0 & $(1,1,1,2,1,1)$ & -4 & 0 \\
\hline & & $\overline{\tilde{h}}$ & $(1,2)$ & 0 & -4 & 0 & 0 & 0 & 0 & 0 & 0 & $(1,1,1,2,1,1)$ & 4 & 0 \\
\hline & & $\xi_{4}$ & $(1,1)$ & 0 & 0 & 0 & 0 & 0 & 0 & 0 & 0 & $(1,1,2,1,2,1)$ & 0 & 0 \\
\hline & & $\xi_{5}$ & $(1,1)$ & 0 & 0 & 0 & 0 & 0 & 0 & 0 & 0 & $(1,1,2,1,2,1)$ & 0 & 0 \\
\hline & & $\phi_{1}$ & $(1,1)$ & 0 & 0 & 0 & 0 & 4 & 0 & 0 & 0 & $(1,1,1,2,1,1)$ & -4 & 0 \\
\hline & & $\phi_{2}$ & $(1,1)$ & 0 & 0 & 0 & 0 & -4 & 0 & 0 & 0 & $(1,1,1,2,1,1)$ & 4 & 0 \\
\hline \multirow[t]{4}{*}{$f$} & \multirow[t]{4}{*}{$\tilde{S}+\zeta$} & $\xi_{6}$ & $(1,1)$ & 0 & 0 & 0 & 0 & 0 & 0 & 0 & 0 & $(1,2,1,1,2,1)$ & 4 & -4 \\
\hline & & $\xi_{7}$ & $(1,1)$ & 0 & 0 & 0 & 0 & 0 & 0 & 0 & 0 & $(1,2,1,1,2,1)$ & -4 & 4 \\
\hline & & $\phi_{3}$ & $(1,1)$ & 0 & 0 & 4 & 0 & 0 & 0 & 0 & 0 & $(2,1,1,1,1,1)$ & 0 & -4 \\
\hline & & $\phi_{4}$ & $(1,1)$ & 0 & 0 & -4 & 0 & 0 & 0 & 0 & 0 & $(2,1,1,1,1,1)$ & 0 & 4 \\
\hline
\end{tabular}


Table 3 The observable matter sectors. All charges are multipled by 4

\begin{tabular}{|c|c|c|c|c|c|c|c|c|c|c|c|c|c|c|}
\hline $\mathrm{F}$ & Sector & Name & $(C, L)$ & $Q_{C}$ & $Q_{L}$ & $Q_{\bar{\eta}^{1}}$ & $Q_{\bar{\eta}^{2}}$ & $Q_{\bar{\eta}^{3}}$ & $Q_{\bar{y} 3,6}$ & $Q_{\bar{y}^{1} \bar{w}^{5}}$ & $Q_{\bar{w}^{2,4}}$ & $S U(2)_{1, \ldots, 6}$ & $Q_{7}$ & $Q_{8}$ \\
\hline \multirow[t]{6}{*}{ f } & \multirow[t]{6}{*}{$b_{1}$} & $Q_{1}$ & $(3,2)$ & 2 & 0 & -2 & 0 & 0 & -2 & 0 & 0 & $(1,1,1,1,1,1)$ & 0 & 0 \\
\hline & & $u_{1}^{c}$ & $(\overline{3}, 1)$ & -2 & -4 & -2 & 0 & 0 & 2 & 0 & 0 & $(1,1,1,1,1,1)$ & 0 & 0 \\
\hline & & $d_{1}^{c}$ & $(\overline{3}, 1)$ & -2 & 4 & -2 & 0 & 0 & -2 & 0 & 0 & $(1,1,1,1,1,1)$ & 0 & 0 \\
\hline & & $L_{1}$ & $(1,2)$ & -6 & 0 & -2 & 0 & 0 & 2 & 0 & 0 & $(1,1,1,1,1,1)$ & 0 & 0 \\
\hline & & $e_{1}^{c}$ & $(1,1)$ & 6 & 4 & -2 & 0 & 0 & 2 & 0 & 0 & $(1,1,1,1,1,1)$ & 0 & 0 \\
\hline & & $N_{1}^{c}$ & $(1,1)$ & 6 & -4 & -2 & 0 & 0 & -2 & 0 & 0 & $(1,1,1,1,1,1)$ & 0 & 0 \\
\hline \multirow[t]{6}{*}{$f$} & \multirow[t]{6}{*}{$b_{2}$} & $Q_{2}$ & $(3,2)$ & 2 & 0 & 0 & -2 & 0 & 0 & 2 & 0 & $(1,1,1,1,1,1)$ & 0 & 0 \\
\hline & & $u_{2}^{c}$ & $(\overline{3}, 1)$ & -2 & -4 & 0 & -2 & 0 & 0 & -2 & 0 & $(1,1,1,1,1,1)$ & 0 & 0 \\
\hline & & $d_{2}^{c}$ & $(\overline{3}, 1)$ & -2 & 4 & 0 & -2 & 0 & 0 & 2 & 0 & $(1,1,1,1,1,1)$ & 0 & 0 \\
\hline & & $L_{2}$ & $(1,2)$ & -6 & 0 & 0 & -2 & 0 & 0 & -2 & 0 & $(1,1,1,1,1,1)$ & 0 & 0 \\
\hline & & $e_{2}^{c}$ & $(1,1)$ & 6 & 4 & 0 & -2 & 0 & 0 & -2 & 0 & $(1,1,1,1,1,1)$ & 0 & 0 \\
\hline & & $N_{2}^{c}$ & $(1,1)$ & 6 & -4 & 0 & -2 & 0 & 0 & 2 & 0 & $(1,1,1,1,1,1)$ & 0 & 0 \\
\hline \multirow[t]{6}{*}{$\mathrm{f}$} & \multirow[t]{6}{*}{$b_{3}$} & $Q_{3}$ & $(3,2)$ & 2 & 0 & 0 & 0 & 2 & 0 & 0 & -2 & $(1,1,1,1,1,1)$ & 0 & 0 \\
\hline & & $u_{3}^{c}$ & $(\overline{3}, 1)$ & -2 & -4 & 0 & 0 & 2 & 0 & 0 & 2 & $(1,1,1,1,1,1)$ & 0 & 0 \\
\hline & & $d_{3}^{c}$ & $(\overline{3}, 2)$ & -2 & 4 & 0 & 0 & 2 & 0 & 0 & -2 & $(1,1,1,1,1,1)$ & 0 & 0 \\
\hline & & $L_{3}$ & $(1,2)$ & -6 & 0 & 0 & 0 & 2 & 0 & 0 & 2 & $(1,1,1,1,1,1)$ & 0 & 0 \\
\hline & & $e_{3}^{c}$ & $(1,1)$ & 6 & 4 & 0 & 0 & 2 & 0 & 0 & 2 & $(1,1,1,1,1,1)$ & 0 & 0 \\
\hline & & $N_{3}^{c}$ & $(1,1)$ & 6 & -4 & 0 & 0 & 2 & 0 & 0 & -2 & $(1,1,1,1,1,1)$ & 0 & 0 \\
\hline
\end{tabular}

Table 4 The hidden sectors. All charges are multipled by 4 and the combination $\zeta=1+b_{1}+b_{2}+b_{3}$ has been used

\begin{tabular}{|c|c|c|c|c|c|c|c|c|c|c|c|c|c|c|}
\hline $\mathrm{F}$ & Sector & Name & $(C, L)$ & $Q_{C}$ & $Q_{L}$ & $Q_{\bar{\eta}^{1}}$ & $Q_{\bar{\eta}^{2}}$ & $Q_{\bar{\eta}^{3}}$ & $Q_{\bar{y}^{3,6}}$ & $Q_{\bar{y}^{1} \bar{w}^{5}}$ & $Q_{\bar{w}^{2,4}}$ & $S U(2)_{1, \ldots, 6}$ & $Q_{7}$ & $Q_{8}$ \\
\hline \multirow[t]{4}{*}{$\mathrm{b}$} & \multirow[t]{4}{*}{$b_{1}+b_{2}+\alpha+\beta$} & $\Phi_{1}^{\alpha \beta}$ & $(1,1)$ & 0 & 0 & 0 & 0 & 0 & 2 & 2 & 0 & $(1,2,1,2,1,1)$ & 0 & 0 \\
\hline & & $\bar{\Phi}_{1}^{\alpha \beta}$ & $(1,1)$ & 0 & 0 & 0 & 0 & 0 & -2 & -2 & 0 & $(1,2,1,2,1,1)$ & 0 & 0 \\
\hline & & $\Phi_{2}^{\alpha \beta}$ & $(1,1)$ & 0 & 0 & 0 & 0 & 0 & -2 & 2 & 0 & $(2,1,2,1,1,1)$ & 0 & 0 \\
\hline & & $\bar{\Phi}_{2}^{\alpha \beta}$ & $(1,1)$ & 0 & 0 & 0 & 0 & 0 & 2 & -2 & 0 & $(2,1,2,1,1,1)$ & 0 & 0 \\
\hline \multirow[t]{2}{*}{$\mathrm{f}$} & \multirow[t]{2}{*}{$\tilde{S}+b_{1}+b_{2}+\alpha+\beta$} & $\tilde{\Phi}_{3}^{\alpha \beta}$ & $(1,1)$ & 0 & 0 & 0 & 0 & 0 & 2 & -2 & 0 & $(2,1,2,1,1,1)$ & 0 & 0 \\
\hline & & $\overline{\tilde{\Phi}}_{3}^{\alpha \beta}$ & $(1,1)$ & 0 & 0 & 0 & 0 & 0 & -2 & 2 & 0 & $(2,1,2,1,1,1)$ & 0 & 0 \\
\hline \multirow[t]{2}{*}{$f$} & \multirow[t]{2}{*}{$\tilde{S}+b_{1}+b_{2}+\alpha+\beta+\zeta$} & $\Phi_{4}^{\alpha \beta}$ & $(1,1)$ & 0 & 0 & 0 & 0 & 0 & 2 & -2 & 0 & $(1,1,1,2,1,2)$ & 0 & 0 \\
\hline & & $\bar{\Phi}_{4}^{\alpha \beta}$ & $(1,1)$ & 0 & 0 & 0 & 0 & 0 & -2 & 2 & 0 & $(1,1,1,2,1,2)$ & 0 & 0 \\
\hline
\end{tabular}

Table $5 S O(10)$ singlets without $\tilde{S}$-partners. All charges are multipled by 4 and the combination $\zeta=1+b_{1}+b_{2}+b_{3}$ has been used

\begin{tabular}{|c|c|c|c|c|c|c|c|c|c|c|c|c|c|c|}
\hline $\mathrm{F}$ & Sector & Name & $(C, L)$ & $Q_{C}$ & $Q_{L}$ & $Q_{\bar{\eta}^{1}}$ & $Q_{\bar{\eta}^{2}}$ & $Q_{\bar{\eta}^{3}}$ & $Q_{\bar{y}^{3,6}}$ & $Q_{\bar{y}} 1 \bar{w}^{5}$ & $Q_{\bar{w}^{2,4}}$ & $S U(2)_{1, \ldots, 6}$ & $Q_{7}$ & $Q_{8}$ \\
\hline \multirow[t]{4}{*}{$\mathrm{b}$} & \multirow[t]{4}{*}{$\alpha+\beta$} & $N_{1}$ & $(1,1)$ & 0 & 0 & -2 & 2 & 0 & 0 & 0 & 0 & $(1,2,1,2,1,1)$ & 0 & 0 \\
\hline & & $\bar{N}_{1}$ & $(1,1)$ & 0 & 0 & 2 & -2 & 0 & 0 & 0 & 0 & $(1,2,1,2,1,1)$ & 0 & 0 \\
\hline & & $\mathrm{N}_{2}$ & $(1,1)$ & 0 & 0 & 2 & -2 & 0 & 0 & 0 & 0 & $(1,2,1,2,1,1)$ & 0 & 0 \\
\hline & & $\bar{N}_{2}$ & $(1,1)$ & 0 & 0 & -2 & 2 & 0 & 0 & 0 & 0 & $(1,2,1,2,1,1)$ & 0 & 0 \\
\hline \multirow[t]{4}{*}{$\mathrm{b}$} & \multirow[t]{4}{*}{$\alpha+\beta+\xi$} & $N_{3}$ & $(1,1)$ & 0 & 0 & -2 & -2 & 0 & 0 & 0 & 0 & $(1,1,1,1,1,2)$ & 4 & 0 \\
\hline & & $\bar{N}_{3}$ & $(1,1)$ & 0 & 0 & 2 & 2 & 0 & 0 & 0 & 0 & $(1,1,1,1,1,2)$ & -4 & 0 \\
\hline & & $N_{4}$ & $(1,1)$ & 0 & 0 & -2 & -2 & 0 & 0 & 0 & 0 & $(1,1,1,1,1,2)$ & 4 & 0 \\
\hline & & $\bar{N}_{4}$ & $(1,1)$ & 0 & 0 & 2 & 2 & 0 & 0 & 0 & 0 & $(1,1,1,1,1,2)$ & -4 & 0 \\
\hline
\end{tabular}


Table $6 S O(10)$ singlets with $\tilde{S}$-partners. All charges are multipled by 4 and the combination $\zeta=1+b_{1}+b_{2}+b_{3}$ has been used

\begin{tabular}{|c|c|c|c|c|c|c|c|c|c|c|c|c|c|c|}
\hline $\mathrm{F}$ & Sector & Name & $(C, L)$ & $Q_{C}$ & $Q_{L}$ & $Q_{\bar{\eta}^{1}}$ & $Q_{\bar{\eta}^{2}}$ & $Q_{\bar{\eta}^{3}}$ & $Q_{\bar{y}^{3}, 6}$ & $Q_{\bar{y}^{1} \bar{w}^{5}}$ & $Q_{\bar{w}^{2,4}}$ & $S U(2)_{1, \ldots, 6}$ & $Q_{7}$ & $Q_{8}$ \\
\hline \multirow[t]{5}{*}{$f$} & \multirow[t]{5}{*}{$b_{1}+2 \gamma$} & $V_{1}$ & $(1,1)$ & 0 & 0 & 0 & -2 & 2 & -2 & 0 & 0 & $(1,1,1,1,1,1)$ & -4 & -4 \\
\hline & & $V_{2}$ & $(1,1)$ & 0 & 0 & 0 & -2 & 2 & -2 & 0 & 0 & $(1,1,1,1,1,1)$ & 4 & 4 \\
\hline & & $V_{3}$ & $(1,1)$ & 0 & 0 & 0 & -2 & 2 & 2 & 0 & 0 & $(1,1,1,1,1,1)$ & -4 & 4 \\
\hline & & $V_{4}$ & $(1,1)$ & 0 & 0 & 0 & -2 & 2 & 2 & 0 & 0 & $(1,1,1,1,1,1)$ & 4 & -4 \\
\hline & & $V_{5}$ & $(1,1)$ & 0 & 0 & 0 & -2 & 2 & -2 & 0 & 0 & $(1,1,1,1,2,2)$ & 0 & 0 \\
\hline \multirow[t]{5}{*}{$\mathrm{f}$} & \multirow[t]{5}{*}{$b_{2}+2 \gamma$} & $V_{6}$ & $(1,1)$ & 0 & 0 & -2 & 0 & 2 & 0 & 2 & 0 & $(1,1,1,1,1,1)$ & -4 & -4 \\
\hline & & $V_{7}$ & $(1,1)$ & 0 & 0 & -2 & 0 & 2 & 0 & 2 & 0 & $(1,1,1,1,1,1)$ & 4 & 4 \\
\hline & & $V_{8}$ & $(1,1)$ & 0 & 0 & -2 & 0 & 2 & 0 & -2 & 0 & $(1,1,1,1,1,1)$ & -4 & 4 \\
\hline & & $V_{9}$ & $(1,1)$ & 0 & 0 & -2 & 0 & 2 & 0 & -2 & 0 & $(1,1,1,1,1,1)$ & 4 & -4 \\
\hline & & $V_{10}$ & $(1,1)$ & 0 & 0 & -2 & 0 & 2 & 0 & 2 & 0 & $(1,1,1,1,2,2)$ & 0 & 0 \\
\hline \multirow[t]{5}{*}{$\mathrm{f}$} & \multirow[t]{5}{*}{$b_{3}+2 \gamma$} & $V_{11}$ & $(1,1)$ & 0 & 0 & -2 & -2 & 0 & 0 & 0 & -2 & $(1,1,1,1,1,1)$ & -4 & -4 \\
\hline & & $V_{12}$ & $(1,1)$ & 0 & 0 & -2 & -2 & 0 & 0 & 0 & -2 & $(1,1,1,1,1,1)$ & 4 & 4 \\
\hline & & $V_{13}$ & $(1,1)$ & 0 & 0 & -2 & -2 & 0 & 0 & 0 & 2 & $(1,1,1,1,1,1)$ & -4 & 4 \\
\hline & & $V_{14}$ & $(1,1)$ & 0 & 0 & -2 & -2 & 0 & 0 & 0 & 2 & $(1,1,1,1,1,1)$ & 4 & -4 \\
\hline & & $V_{15}$ & $(1,1)$ & 0 & 0 & -2 & -2 & 0 & 0 & 0 & -2 & $(1,1,1,1,2,2)$ & 0 & 0 \\
\hline \multirow[t]{2}{*}{$\mathrm{f}$} & \multirow[t]{2}{*}{$b_{1}+2 \gamma+\zeta$} & $V_{16}$ & $(1,1)$ & 0 & 0 & 0 & -2 & 2 & 2 & 0 & 0 & $(1,2,2,1,1,1)$ & 0 & 0 \\
\hline & & $V_{17}$ & $(1,1)$ & 0 & 0 & 0 & -2 & 2 & -2 & 0 & 0 & $(2,1,1,2,1,1)$ & 0 & 0 \\
\hline \multirow[t]{2}{*}{$f$} & \multirow[t]{2}{*}{$b_{2}+2 \gamma+\zeta$} & $V_{18}$ & $(1,1)$ & 0 & 0 & -2 & 0 & 2 & 0 & -2 & 0 & $(1,2,2,1,1,1)$ & 0 & 0 \\
\hline & & $V_{19}$ & $(1,1)$ & 0 & 0 & -2 & 0 & 2 & 0 & 2 & 0 & $(2,1,1,2,1,1)$ & 0 & 0 \\
\hline \multirow[t]{2}{*}{ f } & \multirow[t]{2}{*}{$b_{3}+2 \gamma+\zeta$} & $V_{20}$ & $(1,1)$ & 0 & 0 & -2 & -2 & 0 & 0 & 0 & 2 & $(1,2,2,1,1,1)$ & 0 & 0 \\
\hline & & $V_{21}$ & $(1,1)$ & 0 & 0 & -2 & -2 & 0 & 0 & 0 & -2 & $(2,1,1,2,1,1)$ & 0 & 0 \\
\hline \multirow[t]{3}{*}{ b } & \multirow[t]{3}{*}{$\tilde{S}+b_{1}+2 \gamma$} & $V_{22}$ & $(1,1)$ & 0 & 0 & 0 & 2 & 2 & -2 & 0 & 0 & $(1,1,1,2,1,1)$ & 0 & -4 \\
\hline & & $V_{23}$ & $(1,1)$ & 0 & 0 & 0 & 2 & 2 & 2 & 0 & 0 & $(1,1,1,2,1,1)$ & 0 & 4 \\
\hline & & $V_{24}$ & $(1,1)$ & 0 & 0 & 0 & 2 & -2 & 2 & 0 & 0 & $(1,1,2,1,1,2)$ & 0 & 0 \\
\hline \multirow[t]{3}{*}{$\mathrm{b}$} & \multirow[t]{3}{*}{$\tilde{S}+b_{2}+2 \gamma$} & $V_{25}$ & $(1,1)$ & 0 & 0 & 2 & 0 & 2 & 0 & -2 & 0 & $(1,1,1,2,1,1)$ & 0 & 4 \\
\hline & & $V_{26}$ & $(1,1)$ & 0 & 0 & 2 & 0 & 2 & 0 & 2 & 0 & $(1,1,1,2,1,1)$ & 0 & -4 \\
\hline & & $V_{27}$ & $(1,1)$ & 0 & 0 & 2 & 0 & -2 & 0 & -2 & 0 & $(1,1,2,1,1,2)$ & 0 & 0 \\
\hline \multirow[t]{3}{*}{ b } & \multirow[t]{3}{*}{$\tilde{S}+b_{3}+2 \gamma$} & $V_{28}$ & $(1,1)$ & 0 & 0 & 2 & -2 & 0 & 0 & 0 & 2 & $(1,1,1,2,1,1)$ & 0 & 4 \\
\hline & & $V_{29}$ & $(1,1)$ & 0 & 0 & 2 & -2 & 0 & 0 & 0 & -2 & $(1,1,1,2,1,1)$ & 0 & -4 \\
\hline & & $V_{30}$ & $(1,1)$ & 0 & 0 & 2 & 2 & 0 & 0 & 0 & 2 & $(1,1,2,1,1,2)$ & 0 & 0 \\
\hline \multirow[t]{3}{*}{$\mathrm{b}$} & \multirow[t]{3}{*}{$\tilde{S}+b_{1}+2 \gamma+\zeta$} & $V_{31}$ & $(1,1)$ & 0 & 0 & 0 & 2 & 2 & 2 & 0 & 0 & $(2,1,1,1,1,1)$ & -4 & 0 \\
\hline & & $V_{32}$ & $(1,1)$ & 0 & 0 & 0 & 2 & 2 & -2 & 0 & 0 & $(2,1,1,1,1,1)$ & 4 & 0 \\
\hline & & $V_{33}$ & $(1,1)$ & 0 & 0 & 0 & 2 & -2 & -2 & 0 & 0 & $(1,2,1,1,2,1)$ & 0 & 0 \\
\hline \multirow[t]{3}{*}{$\mathrm{b}$} & \multirow[t]{3}{*}{$\tilde{S}+b_{2}+2 \gamma+\zeta$} & $V_{34}$ & $(1,1)$ & 0 & 0 & 2 & 0 & 2 & 0 & 2 & 0 & $(2,1,1,1,1,1)$ & 4 & 0 \\
\hline & & $V_{35}$ & $(1,1)$ & 0 & 0 & 2 & 0 & 2 & 0 & -2 & 0 & $(2,1,1,1,1,1)$ & -4 & 0 \\
\hline & & $V_{36}$ & $(1,1)$ & 0 & 0 & 2 & 0 & -2 & 0 & 2 & 0 & $(1,2,1,1,2,1)$ & 0 & 0 \\
\hline b & $\tilde{S}+b_{3}+2 \gamma+\zeta$ & $V_{37}$ & $(1,1)$ & 0 & 0 & 2 & -2 & 0 & 0 & 0 & -2 & $(2,1,1,1,1,1)$ & 4 & 0 \\
\hline & & $V_{38}$ & $(1,1)$ & 0 & 0 & 2 & -2 & 0 & 0 & 0 & 2 & $(2,1,1,1,1,1)$ & -4 & 0 \\
\hline & & $V_{39}$ & $(1,1)$ & 0 & 0 & 2 & 2 & 0 & 0 & 0 & -2 & $(1,2,1,1,2,1)$ & 0 & 0 \\
\hline
\end{tabular}


Table 7 Exotic states with $\tilde{S}$-partners (i). All charges are multipled by 4 and the combination $\zeta=1+b_{1}+b_{2}+b_{3}$ has been used

\begin{tabular}{|c|c|c|c|c|c|c|c|c|c|c|c|c|c|c|}
\hline $\mathrm{F}$ & Sector & Name & $(C, L)$ & $Q_{C}$ & $Q_{L}$ & $Q_{\bar{\eta}^{1}}$ & $Q_{\bar{\eta}^{2}}$ & $Q_{\bar{\eta}^{3}}$ & $Q_{\bar{y}^{3,6}}$ & $Q_{\bar{y}^{1} \bar{w}^{5}}$ & $Q_{\bar{w}^{2,4}}$ & $S U(2)_{1, \ldots, 6}$ & $Q_{7}$ & $Q_{8}$ \\
\hline \multirow[t]{2}{*}{$\mathrm{f}$} & \multirow[t]{2}{*}{$b_{2}+\beta$} & $H_{1}$ & $(1,2)$ & 0 & 0 & 0 & 0 & 0 & 2 & 0 & -2 & $(1,1,2,1,1,1)$ & 0 & 0 \\
\hline & & $\bar{H}_{1}$ & $(1,2)$ & 0 & 0 & 0 & 0 & 0 & -2 & 0 & 2 & $(1,1,2,1,1,1)$ & 0 & 0 \\
\hline \multirow[t]{2}{*}{$\mathrm{b}$} & \multirow[t]{2}{*}{$\tilde{S}+b_{2}+\beta$} & $\mathrm{H}_{2}$ & $(1,2)$ & 0 & 0 & 0 & 0 & 0 & 2 & 0 & -2 & $(1,1,1,1,2,1)$ & 0 & 0 \\
\hline & & $\bar{H}_{2}$ & $(1,2)$ & 0 & 0 & 0 & 0 & 0 & -2 & 0 & 2 & $(1,1,1,1,2,1)$ & 0 & 0 \\
\hline \multirow[t]{2}{*}{ b } & $b_{2}+b_{3}$ & $H_{3}$ & $(1,1)$ & -3 & 2 & 1 & 1 & -1 & -2 & 0 & 0 & $(1,1,2,1,1,1)$ & 2 & 2 \\
\hline & $+\beta \pm \gamma$ & $\bar{H}_{3}$ & $(1,1)$ & 3 & -2 & -1 & -1 & 1 & 2 & 0 & 0 & $(1,1,2,1,1,1)$ & -2 & -2 \\
\hline \multirow[t]{4}{*}{$f$} & $\tilde{S}+b_{2}+b_{3}$ & $H_{4}$ & $(1,1)$ & -3 & 2 & 1 & 1 & -1 & -2 & 0 & 0 & $(1,1,1,1,2,1)$ & 2 & 2 \\
\hline & \multirow[t]{3}{*}{$+\beta \pm \gamma$} & $H_{5}$ & $(1,1)$ & -3 & 2 & 1 & 1 & -1 & -2 & 0 & 0 & $(1,1,1,1,1,2)$ & -2 & -2 \\
\hline & & $\bar{H}_{4}$ & $(1,1)$ & 3 & -2 & -1 & -1 & 1 & 2 & 0 & 0 & $(1,1,1,1,2,1)$ & -2 & -2 \\
\hline & & $\bar{H}_{5}$ & $(1,1)$ & 3 & -2 & -1 & -1 & 1 & 2 & 0 & 0 & $(1,1,1,1,1,2)$ & 2 & 2 \\
\hline \multirow[t]{2}{*}{$\mathrm{b}$} & $b_{1}+b_{3}$ & $H_{6}$ & $(1,1)$ & -3 & 2 & 1 & 1 & -1 & 0 & -2 & 0 & $(1,1,1,2,1,1)$ & -2 & -2 \\
\hline & $+\alpha \pm \gamma+\zeta$ & $\bar{H}_{6}$ & $(1,1)$ & 3 & -2 & -1 & -1 & 1 & 0 & 2 & 0 & $(1,1,1,2,1,1)$ & 2 & 2 \\
\hline \multirow[t]{10}{*}{ f } & $\tilde{S}+b_{1}+b_{3}$ & $H_{7}$ & $(\overline{3}, 1)$ & 1 & 2 & 1 & 1 & -1 & 0 & 2 & 0 & $(1,1,1,1,1,1)$ & 2 & -2 \\
\hline & \multirow[t]{9}{*}{$+\alpha \pm \gamma+\zeta$} & $\bar{H}_{7}$ & $(3,1)$ & -1 & -2 & -1 & -1 & 1 & 0 & -2 & 0 & $(1,1,1,1,1,1)$ & -2 & 2 \\
\hline & & $H_{8}$ & $(1,2)$ & -3 & -2 & 1 & 1 & -1 & 0 & -2 & 0 & $(1,1,1,1,1,1)$ & 2 & -2 \\
\hline & & $\bar{H}_{8}$ & $(1,2)$ & 3 & 2 & -1 & -1 & 1 & 0 & 2 & 0 & $(1,1,1,1,1,1)$ & -2 & 2 \\
\hline & & $H_{9}$ & $(1,1)$ & -3 & 2 & -3 & 1 & -1 & 0 & 2 & 0 & $(1,1,1,1,1,1)$ & 2 & -2 \\
\hline & & $\bar{H}_{9}$ & $(1,1)$ & 3 & -2 & 3 & -1 & 1 & 0 & -2 & 0 & $(1,1,1,1,1,1)$ & -2 & 2 \\
\hline & & $H_{10}$ & $(1,1)$ & -3 & 2 & 1 & -3 & -1 & 0 & 2 & 0 & $(1,1,1,1,1,1)$ & 2 & -2 \\
\hline & & $\bar{H}_{10}$ & $(1,1)$ & 3 & -2 & -1 & 3 & 1 & 0 & -2 & 0 & $(1,1,1,1,1,1)$ & -2 & 2 \\
\hline & & $H_{11}$ & $(1,1)$ & -3 & 2 & 1 & 1 & 3 & 0 & 2 & 0 & $(1,1,1,1,1,1)$ & 2 & -2 \\
\hline & & $\bar{H}_{11}$ & $(1,1)$ & 3 & -2 & -1 & -1 & -3 & 0 & -2 & 0 & $(1,1,1,1,1,1)$ & -2 & 2 \\
\hline \multirow[t]{10}{*}{$f$} & \multirow[t]{10}{*}{$b_{3} \pm \gamma$} & $H_{12}$ & $(\overline{3}, 1)$ & 1 & -2 & 1 & 1 & -1 & 0 & 0 & -2 & $(1,1,1,1,1,1)$ & 2 & 2 \\
\hline & & $\bar{H}_{12}$ & $(3,1)$ & -1 & 2 & -1 & -1 & 1 & 0 & 0 & 2 & $(1,1,1,1,1,1)$ & -2 & -2 \\
\hline & & $H_{13}$ & $(1,2)$ & -3 & 2 & 1 & 1 & -1 & 0 & 0 & -2 & $(1,1,1,1,1,1)$ & 2 & 2 \\
\hline & & $\bar{H}_{13}$ & $(1,2)$ & 3 & -2 & -1 & -1 & 1 & 0 & 0 & 2 & $(1,1,1,1,1,1)$ & -2 & -2 \\
\hline & & $H_{14}$ & $(1,1)$ & -3 & -2 & -3 & 1 & -1 & 0 & 0 & -2 & $(1,1,1,1,1,1)$ & 2 & 2 \\
\hline & & $\bar{H}_{14}$ & $(1,1)$ & 3 & 2 & 3 & -1 & 1 & 0 & 0 & 2 & $(1,1,1,1,1,1)$ & -2 & -2 \\
\hline & & $H_{15}$ & $(1,1)$ & -3 & -2 & 1 & -3 & -1 & 0 & 0 & -2 & $(1,1,1,1,1,1)$ & 2 & 2 \\
\hline & & $\bar{H}_{15}$ & $(1,1)$ & 3 & 2 & -1 & 3 & 1 & 0 & 0 & 2 & $(1,1,1,1,1,1)$ & -2 & -2 \\
\hline & & $H_{16}$ & $(1,1)$ & -3 & -2 & 1 & 1 & 3 & 0 & 0 & -2 & $(1,1,1,1,1,1)$ & 2 & 2 \\
\hline & & $\bar{H}_{16}$ & $(1,1)$ & 3 & 2 & -1 & -1 & -3 & 0 & 0 & 2 & $(1,1,1,1,1,1)$ & -2 & -2 \\
\hline \multirow[t]{2}{*}{$\mathrm{b}$} & \multirow[t]{2}{*}{$\tilde{S}+b_{3} \pm \gamma$} & $H_{17}$ & $(1,1)$ & -3 & -2 & 1 & 1 & -1 & 0 & 0 & 2 & $(1,1,1,2,1,1)$ & -2 & 2 \\
\hline & & $\bar{H}_{17}$ & $(1,1)$ & 3 & 2 & -1 & -1 & 1 & 0 & 0 & -2 & $(1,1,1,2,1,1)$ & 2 & -2 \\
\hline \multirow[t]{4}{*}{$\mathrm{b}$} & $b_{1}+b_{2}$ & $H_{18}$ & $(1,1)$ & 3 & 2 & 1 & 1 & 1 & 2 & -2 & 0 & $(1,1,1,1,2,1)$ & 2 & -2 \\
\hline & \multirow[t]{3}{*}{$\alpha+\beta \pm \gamma+\zeta$} & $H_{19}$ & $(1,1)$ & 3 & 2 & 1 & 1 & 1 & 2 & 2 & 0 & $(1,1,1,1,1,2)$ & -2 & 2 \\
\hline & & $H_{20}$ & $(1,1)$ & -3 & -2 & -1 & -1 & -1 & 2 & -2 & 0 & $(1,1,1,1,2,1)$ & -2 & 2 \\
\hline & & $H_{21}$ & $(1,1)$ & -3 & -2 & -1 & -1 & -1 & 2 & 2 & 0 & $(1,1,1,1,1,2)$ & 2 & -2 \\
\hline \multirow[t]{2}{*}{$\mathrm{f}$} & $\tilde{S}+b_{1}+b_{2}$ & $H_{22}$ & $(1,1)$ & 3 & 2 & 1 & 1 & 1 & -2 & 2 & 0 & $(1,1,2,1,1,1)$ & 2 & -2 \\
\hline & $+\alpha+\beta \pm \gamma+\zeta$ & $H_{23}$ & $(1,1)$ & -3 & -2 & -1 & -1 & -1 & -2 & 2 & 0 & $(1,1,2,1,1,1)$ & -2 & 2 \\
\hline
\end{tabular}


Table 8 Exotic states with $\tilde{S}$-partners (ii). All charges are multipled by 4 and the combination $\zeta=1+b_{1}+b_{2}+b_{3}$ has been used

\begin{tabular}{|c|c|c|c|c|c|c|c|c|c|c|c|c|c|c|}
\hline $\mathrm{F}$ & Sector & Name & $(C, L)$ & $Q_{C}$ & $Q_{L}$ & $Q_{\bar{\eta}^{1}}$ & $Q_{\bar{\eta}^{2}}$ & $Q_{\bar{\eta}^{3}}$ & $Q_{\bar{y}^{3}, 6}$ & $Q_{\bar{y}^{1} \bar{w}^{5}}$ & $Q_{\bar{w}^{2,4}}$ & $S U(2)_{1, \ldots, 6}$ & $Q_{7}$ & $Q_{8}$ \\
\hline \multirow[t]{4}{*}{ b } & \multirow[t]{4}{*}{$b_{1}+b_{3}+\alpha+\beta \pm \gamma+\zeta$} & $H_{24}$ & $(1,1)$ & 3 & 2 & 1 & -1 & -1 & 2 & 0 & 2 & $(1,1,1,1,2,1)$ & 2 & -2 \\
\hline & & $H_{25}$ & $(1,1)$ & 3 & 2 & 1 & -1 & -1 & 2 & 0 & -2 & $(1,1,1,1,1,2)$ & -2 & 2 \\
\hline & & $H_{26}$ & $(1,1)$ & -3 & -2 & -1 & 1 & 1 & 2 & 0 & 2 & $(1,1,1,1,2,1)$ & -2 & 2 \\
\hline & & $H_{27}$ & $(1,1)$ & -3 & -2 & -1 & 1 & 1 & 2 & 0 & -2 & $(1,1,1,1,1,2)$ & 2 & -2 \\
\hline \multirow[t]{2}{*}{$f$} & $\tilde{S}+b_{1}+b_{3}$ & $H_{28}$ & $(1,1)$ & 3 & 2 & 1 & -1 & -1 & -2 & 0 & -2 & $(1,1,2,1,1,1)$ & 2 & -2 \\
\hline & $+\alpha+\beta \pm \gamma+\zeta$ & $H_{29}$ & $(1,1)$ & -3 & -2 & -1 & 1 & 1 & -2 & 0 & -2 & $(1,1,2,1,1,1)$ & -2 & 2 \\
\hline \multirow[t]{4}{*}{ b } & \multirow[t]{4}{*}{$b_{2}+b_{3}$} & $H_{30}$ & $(1,1)$ & 3 & 2 & -1 & 1 & -1 & 0 & -2 & 2 & $(1,1,1,1,2,1)$ & 2 & -2 \\
\hline & & $H_{31}$ & $(1,1)$ & 3 & 2 & -1 & 1 & -1 & 0 & -2 & -2 & $(1,1,1,1,1,2)$ & -2 & 2 \\
\hline & & $H_{32}$ & $(1,1)$ & -3 & -2 & 1 & -1 & 1 & 0 & -2 & 2 & $(1,1,1,1,2,1)$ & -2 & 2 \\
\hline & & $H_{33}$ & $(1,1)$ & -3 & -2 & 1 & -1 & 1 & 0 & -2 & -2 & $(1,1,1,1,1,2)$ & 2 & -2 \\
\hline \multirow[t]{2}{*}{ f } & $\tilde{S}+b_{2}+b_{3}$ & $H_{34}$ & $(1,1)$ & 3 & 2 & -1 & 1 & -1 & 0 & 2 & -2 & $(1,1,2,1,1,1)$ & 2 & -2 \\
\hline & $+\alpha+\beta \pm \gamma+\zeta$ & $H_{35}$ & $(1,1)$ & -3 & -2 & 1 & -1 & 1 & 0 & 2 & -2 & $(1,1,2,1,1,1)$ & -2 & 2 \\
\hline
\end{tabular}

Table 9 Exotic spinorials without $\tilde{S}$-partners (i). All charges are multipled by 4 and the combination $\zeta=1+b_{1}+b_{2}+b_{3}$ has been used

\begin{tabular}{|c|c|c|c|c|c|c|c|c|c|c|c|c|c|c|}
\hline$F$ & Sector & Name & $(C, L)$ & $Q_{C}$ & $Q_{L}$ & $Q_{\bar{\eta}^{1}}$ & $Q_{\bar{\eta}^{2}}$ & $Q_{\bar{\eta}^{3}}$ & $Q_{\bar{y}}^{3,6}$ & $Q_{\bar{y}^{1} \bar{w}^{5}}$ & $Q_{\bar{w}^{2,4}}$ & $S U(2)_{1, \ldots, 6}$ & $Q_{7}$ & $Q_{8}$ \\
\hline \multirow[t]{2}{*}{$\mathrm{f}$} & \multirow[t]{2}{*}{$b_{1}+\alpha$} & $H_{36}$ & $(1,2)$ & 0 & 0 & 0 & 0 & 0 & 0 & -2 & 2 & $(2,1,1,1,1,1)$ & 0 & 0 \\
\hline & & $\bar{H}_{36}$ & $(1,2)$ & 0 & 0 & 0 & 0 & 0 & 0 & 2 & -2 & $(2,1,1,1,1,1)$ & 0 & 0 \\
\hline \multirow[t]{2}{*}{$\mathrm{b}$} & $b_{1}+b_{3}$ & $H_{37}$ & $(1,1)$ & -3 & 2 & 1 & 1 & -1 & 0 & -2 & 0 & $(2,1,1,1,1,1)$ & 2 & 2 \\
\hline & $+\alpha \pm \gamma$ & $\bar{H}_{37}$ & $(1,1)$ & 3 & -2 & -1 & -1 & 1 & 0 & 2 & 0 & $(2,1,1,1,1,1)$ & -2 & -2 \\
\hline \multirow[t]{2}{*}{ b } & $\tilde{S}+b_{3}$ & $H_{38}$ & $(1,1)$ & -3 & -2 & 1 & 1 & -1 & 0 & 0 & -2 & $(2,1,1,1,1,1)$ & 2 & -2 \\
\hline & $\pm \gamma+\zeta$ & $\bar{H}_{38}$ & $(1,1)$ & 3 & 2 & -1 & -1 & 1 & 0 & 0 & 2 & $(2,1,1,1,1,1)$ & -2 & 2 \\
\hline \multirow[t]{2}{*}{ b } & $b_{2}+b_{3}$ & $H_{39}$ & $(1,1)$ & -3 & 2 & 1 & 1 & -1 & 2 & 0 & 0 & $(1,2,1,1,1,1)$ & -2 & -2 \\
\hline & $+\beta \pm \gamma+\zeta$ & $\bar{H}_{39}$ & $(1,1)$ & 3 & -2 & -1 & -1 & 1 & -2 & 0 & 0 & $(1,2,1,1,1,1)$ & 2 & 2 \\
\hline \multirow[t]{2}{*}{$f$} & $\tilde{S}+b_{2}+b_{3}$ & $H_{40}$ & $(1,1)$ & 3 & 2 & -1 & 1 & -1 & 0 & 2 & -2 & $(1,2,1,1,1,1)$ & -2 & 2 \\
\hline & $+\alpha+\beta \pm \gamma$ & $H_{41}$ & $(1,1)$ & -3 & -2 & 1 & -1 & 1 & 0 & 2 & -2 & $(1,2,1,1,1,1)$ & 2 & -2 \\
\hline \multirow[t]{2}{*}{$\mathrm{f}$} & $\tilde{S}+b_{1}+b_{3}$ & $H_{42}$ & $(1,1)$ & 3 & 2 & -1 & 1 & -1 & -2 & 0 & -2 & $(1,2,1,1,1,1)$ & -2 & 2 \\
\hline & $+\alpha+\beta \pm \gamma$ & $H_{43}$ & $(1,1)$ & -3 & -2 & 1 & -1 & 1 & -2 & 0 & -2 & $(1,2,1,1,1,1)$ & 2 & -2 \\
\hline \multirow[t]{2}{*}{$f$} & $\tilde{S}+b_{1}+b_{2}$ & $H_{44}$ & $(1,1)$ & 3 & 2 & 1 & 1 & -1 & -2 & 2 & 0 & $(1,2,1,1,1,1)$ & -2 & 2 \\
\hline & $+\alpha+\beta \pm \gamma$ & $H_{45}$ & $(1,1)$ & -3 & -2 & -1 & -1 & 1 & -2 & 2 & 0 & $(1,2,1,1,1,1)$ & 2 & -2 \\
\hline
\end{tabular}




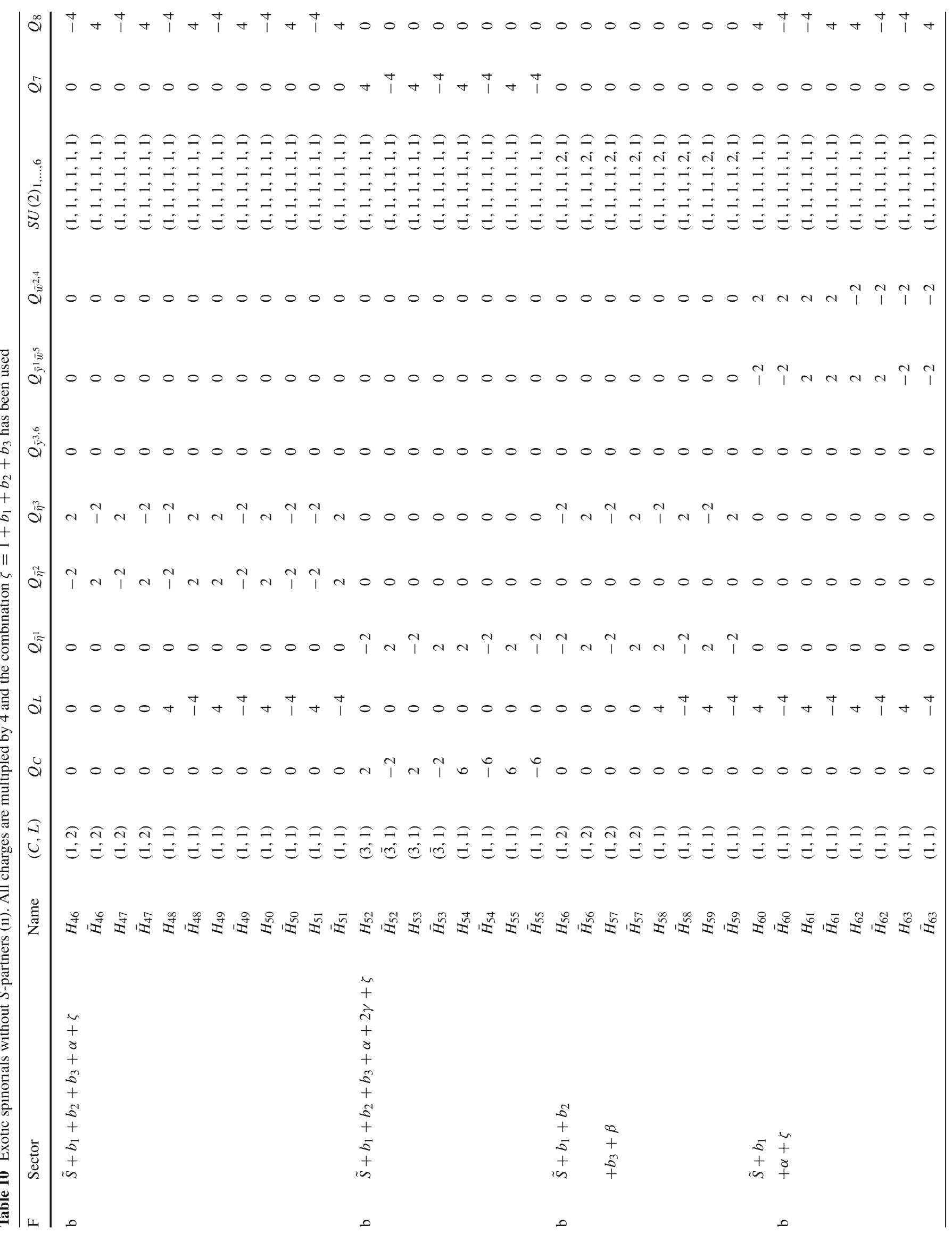




\section{References}

1. I. Antoniadis, C. Bachas, C. Kounnas, Nucl. Phys. B 289, 87 (1987)

2. H. Kawai, D.C. Lewellen, S.H.-H. Tye, Nucl. Phys. B 288, 1 (1987)

3. I. Antoniadis, C. Bachas, Nucl. Phys. B 298, 586 (1988)

4. I. Antoniadis, J. Ellis, J. Hagelin, D.V. Nanopoulos, Phys. Lett. B 231, 65 (1989)

5. A.E. Faraggi, D.V. Nanopoulos, K. Yuan, Nucl. Phys. B 335, 347 (1990)

6. A.E. Faraggi, Phys. Rev. D 46, 3204 (1992)

7. G.B. Cleaver, A.E. Faraggi, D.V. Nanopoulos, Phys. Lett. B 455, 135 (1999)

8. I. Antoniadis, G.K. Leontaris, J. Rizos, Phys. Lett. B 245, 161 (1990)

9. G.K. Leontaris, J. Rizos, Nucl. Phys. B 554, 3 (1999)

10. A.E. Faraggi, Phys. Lett. B 278, 131 (1992)

11. A.E. Faraggi, Nucl. Phys. B 387, 239 (1992)

12. A.E. Faraggi, E. Manno, C.M. Timirgaziu, Eur. Phys. J. C 50, 701 (2007)

13. G.B. Cleaver, A.E. Faraggi, C. Savage, Phys. Rev. D 63, 066001 (2001)

14. G.B. Cleaver, D.J. Clements, A.E. Faraggi, Phys. Rev. D 65, 106003 (2002)

15. B. Assel, C. Christodoulides, A.E. Faraggi, C. Kounnas, J. Rizos, Phys. Lett. B 683, 306 (2010)

16. B. Assel, Nucl. Phys. B 844, 365 (2011)

17. C. Christodoulides, A.E. Faraggi, J. Rizos, Phys. Lett. B 702, 81 (2011)

18. L. Bernard et al., Nucl. Phys. B 868, 1 (2013)

19. A.E. Faraggi, J. Rizos, H. Sonmez, Nucl. Phys. B 886, 202 (2014)

20. H. Sonmez, Phys. Rev. D 93, 125002 (2016)

21. A.E. Faraggi, J. Rizos, H. Sonmez, Nucl. Phys. B 927, 1 (2018)

22. A.E. Faraggi, G. Harries, J. Rizos, Nucl. Phys. B 936, 472 (2018)

23. See e.g.: C. Kounnas, N. Toumbas, PoS Corfu, 2012083 (2013). arXiv:1305.2809, and references therein

24. A.E. Faraggi, Phys. Lett. B 326, 62 (1994)

25. E. Kiritsis, C. Kounnas, Nucl. Phys. B 503, 117 (1997)

26. A.E. Faraggi, S. Forste, C. Timirgaziu, JHEP 0608, 057 (2006)

27. P. Athanasopoulos, A.E. Faraggi, S.G. Nibbelink, V.M. Mehta, JHEP 1604, 038 (2016)

28. See e.g.: A. Taormina, K. Wendland, arXiv:1908.03148

29. L.J. Dixon, J.A. Harvey, Nucl. Phys. B 274, 93 (1986)

30. L. Alvarez-Gaume, P.H. Ginsparg, G.W. Moore, C. Vafa, Phys. Lett. B 171, 155 (1986)

31. P.H. Ginsparg, C. Vafa, Nucl. Phys. B 289, 414 (1986)

32. H. Itoyama, T.R. Taylor, Phys. Lett. B 186, 129 (1987)

33. H. Kawai, D.C. Lewellen, S.H.H. Tye, Phys. Rev. D 34, 3794 (1986)

34. K.R. Dienes, Phys. Rev. Lett. 65, 1979 (1990)

35. K.R. Dienes, Phys. Rev. D 42, 2004 (1990)

36. S. Abel, K.R. Dienes, Phys. Rev. D 91, 126014 (2015)

37. M. Blaszczyk, S.G. Nibbelink, O. Loukas, F. Ruehle, JHEP 1510, 166 (2015)

38. S.G. Nibbelink, E. Parr, Phys. Rev. D 94, 041704 (2016)

39. I. Florakis, J. Rizos, Nucl. Phys. B 913, 495 (2016)

40. I. Florakis, arXiv: 1611.10323

41. S.G. Nibbelink et al., arXiv: 1710.09237

42. S. Abel, K.R. Dienes, E. Mavroudi, Phys. Rev. D 97, 126017 (2018)

43. T. Coudarchet, H. Partouche, Nucl. Phys. B 933, 134 (2018)
44. A. Abel, E. Dudas, D. Lewis, H. Partouche, arXiv:1812.09714

45. H. Itoyama, S. Nakajima, arXiv:1905.10745

46. M. McGuigan, arXiv:1907.01944

47. A.E. Faraggi, M. Tsulaia, Phys. Lett. B 683, 314 (2010)

48. B. Aaronson, S. Abel, E. Mavroudi, Phys. Rev. D 95, 106001 (2017)

49. J.M. Ashfaque, P. Athanasopoulos, A.E. Faraggi, H. Sonmez, Eur. Phys. J. C 76, 208 (2016)

50. A.E. Faraggi, Eur. Phys. J. C 79, 703 (2019)

51. C. Kounnas, Fortsch. Phys. 56, 1143 (2008)

52. I. Florakis, C. Kounnas, Nucl. Phys. B 820, 237 (2009)

53. I. Florakis, C. Kounnas, N. Toumbas, Nucl. Phys. B 834, 273 (2010)

54. N.V. Krasnikov, Phys. Lett. B 193, 37 (1987)

55. L.J. Dixon, Supersymmetry breaking in string theory, in The Rice Meeting: Proceedings ed. by B. Bonner, H. Miettinen (World Scientific, Singapore, 1990)

56. G.B. Cleaver, A.E. Faraggi, E. Manno, C. Timirgaziu, Phys. Rev. D 78, 046009 (2008)

57. G.B. Cleaver, A.E. Faraggi, S.E.M. Nooij, Nucl. Phys. B 672, 64 (2003)

58. A.E. Faraggi, H. Sonmez, Phys. Rev. D 91, 066006 (2015)

59. A. Gregori, C. Kounnas, J. Rizos, Nucl. Phys. B 549, 16 (1999)

60. A.E. Faraggi, C. Kounnas, S.E.M Nooij, J. Rizos, arXiv:hep-th/0311058

61. A.E. Faraggi, C. Kounnas, S.E.M. Nooij, J. Rizos, Nucl. Phys. B 695, 41 (2004)

62. A.E. Faraggi, C. Kounnas, J. Rizos, Phys. Lett. B 648, 84 (2007)

63. A.E. Faraggi, C. Kounnas, J. Rizos, Nucl. Phys. B 774, 208 (2007)

64. A.E. Faraggi, C. Kounnas, J. Rizos, Nucl. Phys. B 799, 19 (2008)

65. T. Catelin-Julian, A.E. Faraggi, C. Kounnas, J. Rizos, Nucl. Phys. B 812, 103 (2009)

66. C. Angelantonj, A.E. Faraggi, M. Tsulaia, JHEP 1007, 4 (2010)

67. A.E. Faraggi, D.V. Nanopoulos, Phys. Rev. D 48, 3288 (1993)

68. A.E. Faraggi, Int. J. Mod. Phys. A 14, 1663 (1999)

69. A.E. Faraggi, Nucl. Phys. B 428, 111 (1994)

70. A.E. Faraggi, Phys. Lett. B 520, 337 (2001)

71. A.E. Faraggi, Phys. Lett. B 274, 47 (1992)

72. A.E. Faraggi, Phys. Rev. D 47, 5021 (1993)

73. A.E. Faraggi, Nucl. Phys. B 728, 83 (2005)

74. A.E. Faraggi, Nucl. Phys. B 407, 57 (1993)

75. A.E. Faraggi, Eur. Phys. J. C 49, 803 (2007)

76. A.E. Faraggi, I. Florakis, T. Mohaupt, M. Tsulaia, Nucl. Phys. B 848, 332 (2011)

77. G. Cleaver, A.E. Faraggi, Int. J. Mod. Phys. A 14, 2335 (1999)

78. M.B. Green, J.H. Schwarz, Phys. Lett. B 149, 117 (1984)

79. J.J. Atick, L.J. Dixon, A. Sen, Nucl. Phys. B 292, 109 (1987)

80. L.J. Dixon, V. Kaplunovsky, J. Louis, Nucl. Phys. B 329, 27 (1990)

81. S. Groot Nibbelink, M. Trapletti, M. Walter, Phys. Lett. B 652, 124 (2007)

82. S. Groot Nibbelink, T.W. Ha, M. Trapletti, Phys. Rev. D 77, 026002 (2008)

83. S.G. Nibbelink, D. Klevers, F. Ploger, M. Trapletti, P.K.S. Vaundrevange, JHEP 0804, 60 (2008)

84. J. Bagger, D. Nemeschansky, N. Seiberg, S. Yankielowicz, Nucl. Phys. B 289, 53 (1987)

85. D. Chang, A. Kumar, Phys. Rev. D 38, 1893 (1988)

86. D. Chang, A. Kumar, Phys. Rev. D 38, 3734 (1988)

87. M. Dine, N. Seiberg, E. Witten, Nucl. Phys. B 289, 589 (1987)

88. G. Cleaver et al., Eur. Phys. J. C 71, 1842 (2011) 